\title{
Angst in der Ratgeberliteratur
}

\section{Eine kulturanalytische linguistische Studie}

\begin{abstract}
Die Studie untersucht, wie in Ratgebern über Angst geschrieben wird und wie sich dies mit der Zeit verändert. Ihr liegen 18 deutschsprachige Ratgeber gegen Angst zugrunde, die zwischen 1985 und 2017 erschienen sind. Mit text-, diskurs- und korpuslinguistischen Methoden werden in diesem Korpus sprachliche Muster ermittelt und ausgehend von Prämissen der kulturanalytischen Linguistik daraufhin interpretiert, welche Perspektiven auf und Umgangsweisen mit Angst sie nahelegen. Vorgestellt und gedeutet werden Muster im Aufbau und hinsichtlich der behandelten Themen, des Weiteren Muster in der Bestimmung von Angst, in der Formulierung von Zielen für die Adressierten sowie in konkreten Ratschlägen bzw. Instruktionen. Die Untersuchung ergibt, dass Angst in den Ratgebern gesamthaft gesehen fast nur als individuelle und kaum als kollektive Emotion erscheint. Als solche kann und soll Angst nicht etwa als menschliche Existenzbedingung ertragen, sondern aktiv reduziert oder mittels anderer Gefühle entkräftet werden. Dazu ist laut den Ratgebern nicht unbedingt die persönliche Hilfe von anderen notwendig, sondern dies kann und soll auch durch Selbststeuerung erfolgen. Die Ratgeber oszillieren damit zwischen der Ermächtigung der/des Einzelnen zur Einwirkung auf die eigenen Gefühle und ihrer/seiner Belastung mit der Verantwortung, die eigenen Gefühle so zu regulieren, dass sie der sozialen Norm entsprechen.
\end{abstract}

The study examines how anxiety (Angst) is written about in guidebooks and how this changes over time. It is based on 18 German guidebooks against anxiety which have been published between 1985 and 2017. Using methods from text, discourse, and corpus linguistics, the study identifies linguistic patterns in this corpus. Starting from premises of cultural linguistics, these patterns or regularities are interpreted with regard to the question which perspectives on anxiety and which ways of dealing with anxiety they suggest. The study identifies and interprets patterns in the composition of the guidebooks, in the topics addressed, in the definition of anxiety, in the formulation of goals for the addressees and in advice that the books give. It can be shown that anxiety (Angst) is presented almost exclusively as an individual and hardly as a collective emotion in the guidebooks. The books suggest that anxiety does not have to be endured as a condition of human existence but that it can actively be reduced or invalidated by other feelings. According to the books, this does not necessarily 
require the personal help of others, but can and should be achieved by selfmanagement. The guidebooks thus oscillate between empowering the individual to influence his/her own feelings and burdening him/her with the responsibility to regulate his/her own feelings so that they conform to the social norm.

\section{Einleitung}

Wie wird in deutschsprachigen Ratgebern der letzten Jahrzehnte über Angst geschrieben, und wie verändert sich dies? Welche Perspektiven auf und Umgangsweisen mit Angst werden dadurch nahegelegt?

Das sind die Fragen, auf die ich in diesem Beitrag Antworten geben möchte. Motiviert sind die Fragen durch die große Bedeutung, die sogenannten Angststörungen einerseits und der Ratgeberliteratur andererseits gegenwärtig zukommen: Nach einer jüngeren Studie des Robert Koch-Instituts hatten mehr als 15\% der 18- bis 79-jährigen Bevölkerung Deutschlands innerhalb eines Jahres eine Angststörung (Robert Koch-Institut 2015: 113); und nach meiner eigenen Stichprobe verzeichnet die Deutsche Nationalbibliothek für die Publikationsjahre 2010 bis 2019 in ihrem Onlinekatalog 23'698 Titel mit dem Schlagwort Ratgeber, wovon 279 einen Bezug zu Angst aufweisen (Deutsche Nationalbibliothek [2019]). Auch wenn diese Befunde äußerst punktuell sind und ihnen bereits sprachgebundene Kategorisierungen zugrunde liegen, bilden sie deutliche Anhaltspunkte dafür, dass Ratgeber gegen Angst als Phänomen an der Schnittstelle von Angst und Ratgeberliteratur kulturell nicht unbedeutend sind und dass die Produktion und Rezeption von Ratgebern ein nicht unwesentliches Element unseres Umgangs mit Angst bilden. Interessiert man sich für Letzteren, liegt folglich eine Beschäftigung mit Ratgebern zum Thema Angst nahe.

Wenn ich mich dementsprechend in dieser Studie mit Angst-Ratgebern beschäftige, dann geschieht dies aus linguistisch-kulturanalytischer Perspektive, wie die eingangs genannten Fragen schon andeuten: Ich gehe davon aus, dass Ratgeber gegen Angst kulturell verbreitete Vorstellungen davon, was Angst ist und was man mit ihr tun kann oder soll, zum Ausdruck bringen und beeinflussen; und ich nehme an, dass eine Analyse des Sprachgebrauchs in solchen Ratgebern es ermöglicht, diese Vorstellungen zu erschließen.

Um dies tun zu können, gehe ich in Teil 2 zunächst auf die relevante Forschung für meine Untersuchung ein. Wie die Übersicht ergibt, hat sich die Linguistik der Verbindung von Sprache und Angst, aber auch der Ratgeberliteratur bisher mit anderen als den hier verfolgten Interessen genähert. In Teil 3 erörtere ich die kulturanalytischen Annahmen genauer, die der Untersuchung voraus- 
gehen, stelle dann das Korpus der Untersuchung vor, das 18 deutschsprachige Ratgeber gegen Angst aus der Zeit von 1985 bis 2017 umfasst, und erläutere anschließend die Methoden, d. h. die text-, diskurs- und korpuslinguistischen Instrumente, die in der Untersuchung verwendet werden. Anschließend präsentiere ich in Teil 4 die sprachlichen Muster, die sich in den ausgewählten Ratgebern zeigen - Muster im Aufbau und hinsichtlich der behandelten Themen, in der Bestimmung von Angst, in der Formulierung von Zielen für die Adressierten sowie in konkreten Ratschlägen bzw. Instruktionen. Die aufgefundenen Muster interpretiere ich insbesondere daraufhin, welche Perspektiven auf und Umgangsweisen mit Angst sie nahelegen. Schließlich versuche ich im Fazit, die zentralen Ergebnisse noch weiter auszudeuten, und werfe einige Fragen für die weitere Forschung auf.

\section{Forschungsstand}

Die relevanteste Forschungsliteratur für diese Studie betrifft Fragen und Gegenstände, die relativ weit weg von den hier behandelten liegen. Veröffentlichungen zur Versprachlichung von Angst in der deutschsprachigen zeitgenössischen Ratgeberliteratur gibt es, soweit ich sehe, noch nicht, und die vorliegenden Publikationen zu den Bereichen Sprache und Angst einerseits und Ratgeberliteratur andererseits sind für meine Untersuchung vergleichsweise wenig ergiebig.

So hat sich die linguistische, aber auch literatur- und sozialwissenschaftliche Forschung zu Sprache und Angst bisher beispielsweise mit Angst als Faktor im (Fremd)Spracherwerb und -gebrauch beschäftigt (vgl. Gkonou, Daubney \& Dewaele [2017], um nur ein aussagekräftiges Beispiel für solche Untersuchungen von language anxiety oder speech anxiety zu nennen); auch die Konstruktion von Angst in politischen und anderen Diskursen hat Aufmerksamkeit gefunden (für eine idealtypische Publikation dieser Art vgl. Wodak 2015); des Weiteren sind Angst-Appelle (fear appeals) in Argumentationen und als Persuasionsmittel analysiert worden (vgl. beispielhaft Walton 2000); und auch die literarische Reflexion auf bzw. Präsentation von Angst ist zum Thema gemacht geworden (vgl. exemplarisch Guntersdorfer 2013). Näher an meiner Studie liegen lexikologisch-phraseologische, semantische bzw. kontrastive Arbeiten zum Angstwortschatz im Deutschen und ggf. in weiteren Sprachen (vgl. z. B. Wandruszka 1950/1981; Bergenholtz 1980; Dobrovol’skij 1995; Cieślarová 2012; Filatkina 2016), Untersuchungen zur begrifflichen Erfassung und Thematisierung von Angst in speziellen Diskursausschnitten (vgl. z. B. Filatkina 2015; Georgi 2018) sowie Analysen der Formulierung und interaktiven Bearbeitung von Ängsten im 
Gespräch (vgl. z. B. Günthner 2006; Gülich, Lindemann \& Schöndienst 2010; Lindemann 2012; Knerich 2013; vgl. auch das frühe Plädoyer für entsprechende linguistische Untersuchungen Schwarz 1988), insofern sie alle Möglichkeiten der Versprachlichung von Angst in der deutschen Sprache betreffen. An die genannten Veröffentlichungen grenzen wiederum solche zum Sprechen und Schreiben über psychische Auffälligkeiten, Belastungen bzw. Krankheiten jenseits von Angststörungen (vgl. z. B. Schuster 2010; Scheidt et al. 2015; Gredel 2016; Iakushevic 2018) sowie zum Verhältnis von Sprache und Emotionen generell an (vgl. z. B. Athanasiadou \& Tabakowska 1998; Fiehler 2001; SchwarzFriesel 2007/2013). In diesem Zusammenhang sind schließlich auch Publikationen zur Kulturgeschichte der Angst erwähnenswert, die im Rahmen der geschichtswissenschaftlichen Erforschung der history of emotions entstanden sind, wobei diese Publikationen in der Regel weniger sprachbezogen sind (vgl. z. B. Bourke 2005/2006; Laffan \& Weiss 2012; mehrere Beiträge in Plamper \& Lazier 2012). Aktuelle Buchprojekte (vgl. diesen Band sowie Knuchel \& Luth [erscheint]) deuten erfreulicherweise darauf hin, dass das linguistische und interdisziplinäre Interesse am Themenbereich Sprache und Angst derzeit zunimmt.

Auch die bisherige sprachwissenschaftliche Forschung zur Ratgeberliteratur berührt sich nur wenig mit meinem Beitrag. Zwar sind deutschsprachige Ratgeber immer wieder als Material für Untersuchungen herangezogen worden, größtenteils handelt es sich dabei jedoch um Sprach- und Kommunikationsratgeber (z. B. Bremerich-Vos 1991; Antos 1996; Schneider 2005; Kessel 2009) bzw. um ihre historischen Vorgänger wie Anstandsbücher, Briefsteller usw. (z. B. Nickisch 1969; Ettl 1984; Linke 1996; Schröter 2011). Diese Texte sind vor allem auf die in ihnen enthaltenen sprachlichen und kommunikativen Normen hin ausgewertet worden - sei es, um Sprach- bzw. Kommunikationsreflexion zu erforschen, Spracheinstellungen bzw. Sprachbewusstsein zu rekonstruieren, Rückschlüsse auf die sprachliche und kommunikative Praxis zu ziehen, oder auch, um sich kritisch mit nicht-linguistischen Normierungen von Sprache und Kommunikation auseinanderzusetzen. Analysen von Ratgebern $\mathrm{zu}$ anderen Themen und der Praktik des Ratgebens an sich sind demgegenüber bislang selten (zu den Ausnahmen gehören z. B. Niemann 2018, der ebenfalls auf dieses Forschungsdesiderat hinweist; Ott \& Kiesendahl 2019; Niemann 2020). Auch die linguistische Aufmerksamkeit für Ratgeber und das Ratgeben scheint sich allerdings momentan $\mathrm{zu}$ verstärken. ${ }^{1}$

1 So veranstalteten etwa Mathilde Hennig und Robert Niemann im Herbst 2020 eine Tagung mit dem Titel Ratgebepraxis in der spätmodernen Gesellschaft. Sprache, Normierung, Wissen. 


\section{Theoretische Prämissen, Korpus und Methoden}

Ich arbeite in dieser Studie mit zentralen theoretischen Prämissen der kulturanalytischen Linguistik (vgl. dazu Linke 2011: 26-32, 37-41; Schröter 2014: 3031, 36-37; Schröter, Tienken \& Ilg 2019: 5-10). Dazu gehört zunächst ein grundsätzlich konstruktivistisches Verständnis des Verhältnisses von Sprachgebrauch, Kognition und kultureller Wirklichkeit, d.h. die Annahme, dass Sprachgebrauch die sonstige kulturelle Wirklichkeit nicht nur reflektiert bzw. repräsentiert, sondern dass er sie normalerweise auch konstituiert, modifiziert oder stabilisiert, indem er unsere Wahrnehmungen, Kategorisierungen, Überzeugungen, Bewertungen und natürlich auch Gefühle und damit potenziell auch unser Verhalten und Handeln beeinflusst. „Sprachliches und anderweitig Kulturelles [stehen]“ demnach ,in einem Verhältnis der gegenseitigen Hervorbringung“ (Schröter, Tienken \& Ilg 2019: 6). Hieran schließt eine weitere Grundüberzeugung der kulturanalytischen Linguistik an: die Vorstellung, dass Sprachgebrauch und insbesondere Muster des Sprachgebrauchs insofern kulturell signifikant sein können, als sie Aufschluss über weit verbreitete, wirkmächtige Wahrnehmungen, Kategorisierungen, Überzeugungen, Bewertungen und Gefühle geben können. Methodisch zentral ist für die kulturanalytische Linguistik demgemäß das „Erkennen von Mustern“ auf der „traditionell sogenannten sprachlichen ,Oberfläche““ und die „Entwicklung kulturbezogener Deutungen bzw. die Rekonstruktion kulturellen Sinns aus diesen Mustern“ (Schröter, Tienken \& Ilg 2019: 6). Zu den Deutungen gelangt man am einfachsten durch systematisches Fragen nach den möglichen Ursachen und Folgen der beobachteten Sprachgebrauchsmuster. Dass es sich bei den Deutungen nicht um Gewissheiten handeln kann, sondern lediglich um Überlegungen mit hypothetischem Charakter, deren Plausibilität von den Ergebnissen weiterer Untersuchungen abhängt, stellt aus der Sicht der kulturanalytischen Linguistik kein Manko dar, sondern ist dezidiert so vorgesehen. Mit den beiden genannten Prämissen dem konstruktivistischen Verständnis von Sprachgebrauch und der Auffassung von Sprachgebrauchsmustern als kulturell signifikant - ähnelt die kulturanalytische Linguistik einigen anderen sprachwissenschaftlichen Forschungsrichtungen, am stärksten der linguistischen Diskursanalyse. In diskurslinguistischen Arbeiten werden die Prämissen allerdings in der Regel auf Diskurse und nicht auf Sprachgebrauch generell sowie der Tendenz nach auf gesellschaftliches Wissen und nicht auf Kulturelles allgemein bezogen.

Für meine Auseinandersetzung mit Ratgebern gegen Angst bedeuten die vorgestellten Annahmen, dass ich davon ausgehe, dass Angst zwar in Teilen eine universelle menschliche Empfindung ist, dass aber das Erleben dieser „Ba- 
sisemotion“ (Schwarz-Friesel 2007/2013: 250), deren Auslöser und Ausdruck, deren begriffliche Einordnung, Reflexion und Beurteilung sowie innerliche und äußerliche Bearbeitung kulturspezifisch sind. Ich nehme weiter an, dass das Kulturspezifische daran wesentlich durch das Sprechen und Schreiben über Angst geprägt ist und dass es sich durch die Auffindung und Ausdeutung sprachlich-kommunikativer Muster erschließen lässt. Was ich hier als innerliche und äußerliche Bearbeitung von Angst beschrieben habe, lässt sich mit Arlie R. Hochschild auch als „emotion work“ fassen, d. h. als ,act of trying to change in degree or quality an emotion or feeling“ (Hochschild 1979: 561, vgl. 561-563). Hochschild argumentiert, dass wir unsere Gefühle bearbeiten oder managen, indem wir versuchen, sie mit kulturspezifischen Gefühlsregeln für bestimmte soziale Situationen in Einklang zu bringen. Die untersuchten Ratgeber können Hochschild folgend als Kodizes solcher „feeling rules“ verstanden werden, also als Sammlungen ausdrücklich formulierter „social guidelines that direct how we want to try to feel“ (Hochschild 1979: 563, vgl. 563-566). Hochschilds soziologischer theoretischer Ansatz ist von Reinhard Fiehler im deutschsprachigen Raum zu linguistischen Zwecken adaptiert worden. Auch Fiehler geht von „Emotionsregulation“ auf Basis von „Emotionsregeln“ aus, wobei er Letztere um „Manifestationsregeln“, „Korrespondenzregeln“ und „Kodierungsregeln“ ergänzt, die in diesem Zusammenhang allerdings kaum relevant sind (Fiehler 2001: 1427-1428). Statt von ausdrücklich formulierten Hochschild'schen feeling rules könnte man im Falle der Angst-Ratgeber auch von expliziter „emotionology“ im Sinne Peter N. und Carol Z. Stearns' sprechen, d. h. von „attitudes or standards that a society, or a definable group within a society, maintains toward basic emotions and their appropriate expression; ways that institutions reflect and encourage these attitudes in human conduct" (Stearns \& Stearns 1985: 813). Stearns und Stearns weisen aus der Perspektive der Geschichtswissenschaft auf die methodische Notwendigkeit hin, zwischen kulturspezifischen Standards, Werten oder Idealen für Emotionen und dem tatsächlichen individuellen oder gruppengebundenen emotionalen Erleben zu unterscheiden, wobei auch sie unter normalen Umständen einen Einfluss Ersterer auf Letztere annehmen. Besonders bedenkenswert für die vorliegende Untersuchung ist ihre Annahme, dass „[e]motionology surely contributes to definitions of deviance“ (Stearns \& Stearns 1985: 832).

Mein Korpus setzt sich aus insgesamt 18 Ratgebern gegen Angst zusammen. ${ }^{2}$ Es handelt sich dabei um Bücher, die das Stichwort Angst(-) im Titel tra-

2 Ich danke Anne Diehr, Daniel Pfurtscheller, Renate Schröter und Sebastian Thome herzlich für ihre Hilfe bei der Zusammenstellung des Korpus. 
gen, die in Bibliothekskatalogen als Ratgeber klassifiziert sind und als deren Hauptziel sich bei der Einsichtnahme und ersten Lektüre erweist, der Leserschaft zu einem veränderten Umgang mit Angst zu verhelfen. Damit liegt meiner Auswahl eine Auffassung von Ratgeber zugrunde, die weitgehend dem alltagssprachlichen Verständnis als „Buch o. Ä., in dem Anleitungen, Tipps o. Ä. für die Praxis auf einem bestimmten Gebiet enthalten sind“, entspricht (Bibliographisches Institut 2019b). Zwar sind deutschsprachige Ratgeber zum Thema Angst vereinzelt bereits in den 1970er Jahren erschienen, eine Serie solcher Ratgeber lässt sich aber erstmals für die 1980er und 1990er Jahre zusammenstellen. Dementsprechend stammen sechs Ratgeber meines Korpus aus den 1980er und 1990er Jahren, sechs aus den 2000er Jahren und sechs aus den 2010er Jahren. Abgesehen von zwei Fällen - einer deutschen Adaption eines englischen Textes und einer Zweitauflage, die nur ein Jahr nach der ersten Auflage erschienen ist - wurde jeweils die erste Auflage der Ratgeber verwendet. Bei der Auswahl habe ich im Sinne der Vergleichbarkeit darauf geachtet, dass die Ratgeber nicht auf eine Angst spezialisiert sind, die sich auf ein einzelnes Objekt bezieht. Ratgeber speziell gegen Angst vorm Fliegen, vor Prüfungen, vor dem Zahnarzt u. Ä. sind folglich nicht ins Korpus aufgenommen worden. Viele Ratgeber kombinieren das Thema Angst allerdings mit einem weiteren Thema, etwa Depression, Panik oder Sorgen. Solche Ratgeber wie auch Ratgeber, die allgemeine, sogenannte generalisierte Angststörungen behandeln, sind hingegen ins Korpus eingegangen, weil die Materialbasis sonst zu schmal geworden wäre. Nicht ausgeschlossen habe ich des Weiteren Übersetzungen aus dem Englischen, da diese in den untersuchten Dekaden eine wichtige Rolle auf dem Markt der deutschsprachigen Angst-Ratgeber spielen. Während die thematische Spezialisierung und die Sprache, in der sie zuerst erschienen sind, bei allen Ratgebern zweifelsfrei festgestellt werden kann, ist deren Zuordnung zu einer Richtung der Psychotherapie oft schwierig und mit Unklarheiten verbunden. Die Gründe dafür sind, dass viele Verfassende ihren Ratgeber nicht explizit innerhalb des Spektrums psychotherapeutischer Ansätze verorten, dass andere wiederum erwähnen, in ihrem Ratgeber verschiedene Ansätze kombinieren zu wollen, und dass die „Vielfalt [psycho]therapeutischer Modelle, Schulen und Subschulen mittlerweile“ generell „selbst für die im Feld langjährig Tätigen schwer überschaubar [ist]“" (Slunecko 2009/2017: 14).

Aus dem Blickwinkel der linguistischen Kulturanalyse ist bereits das Faktum aufschlussreich, dass Angst-Ratgeber erst seit den 1980er Jahren reihenweise erscheinen. Dies könnte ein Ausdruck dessen sein, dass sich in den 1980er Jahren die Zahl der Menschen, die viel Angst haben, erhöht hat. Doch auch andere Ursachen sind denkbar - etwa, dass bislang disparat kategorisierte 
psychische Phänomene neu unter dem Begriff Angst zusammengefasst werden, dass Angst weniger als bisher akzeptiert wird, dass sie stärker als zuvor als behandel- und reduzierbar gilt oder dass die Kommunikation über Angst weniger tabuisiert ist als in früheren Dekaden. Es könnte zudem sein, dass sich in den 1980er Jahren der Markt der Ratgeberliteratur (nach US-amerikanischem Vorbild?) soweit vergrößert hat, dass nun auch Angst zum Kreis der Themen gehört, die von Ratgebern bearbeitet werden.

Um nicht nur aus der Existenz, sondern auch aus dem Sprachgebrauch in Ratgebern gegen Angst Schlüsse dieser Art ziehen zu können, habe ich die 18 Ratgeber des Korpus mit einer Mischung aus qualitativen und quantitativen Methoden untersucht. Zum Einsatz gekommen sind Instrumente der Text-, Diskurs- und Korpuslinguistik. Um die Ratgeber auch korpuslinguistisch untersuchen zu können, sind ihre inhaltlich relevanten Teile unter Wahrung des Urheberrechts digitalisiert worden. Nicht einbezogen worden sind z. B. Kopf- oder Fußzeilen, Seitenzahlen, Marginalien, Adresslisten, Literaturverzeichnisse, Register, Verlagswerbung und dergleichen. Um diachrone Veränderungen verfolgen zu können, habe ich die digitalisierten Ratgebertexte nicht nur zu einem Gesamtkorpus zusammengefügt, sondern zusätzlich drei Teilkorpora gebildet, die die Texte der 1980er und 1990er Jahre, der 2000er Jahren oder der 2010er Jahre enthalten. Für die korpuslinguistischen Analysen ist die Freeware \#LancsBox verwendet worden (Brezina, Timperley \& McEnery 2018; vgl. Brezina, McEnery \& Wattam 2015). Die Präsentation der Ergebnisse im nächsten Teil mag den Eindruck erwecken, dass ich mit qualitativen Methoden Hypothesen generiert und diese dann korpuslinguistisch überprüft habe. Tatsächlich ist die Analyse der digitalen Korpora aber zunächst der qualitativen Analyse vorausgegangen und dann im Anschluss an diese weitergeführt worden. Für alle vorgestellten Muster gilt, dass es in einzelnen Ratgebern auch Abweichungen gibt, die jedoch aus Platzgründen nicht immer separat erwähnt werden können. Beschrieben wird im Folgenden also das, was mehrheitlich der Fall ist, nicht das, was ausnahmslos gilt.

\section{Ergebnisse}

\subsection{Aufbau und Themen}

Sieht man die Inhaltsverzeichnisse und den Inhalt der Ratgeber gegen Angst auf wiederkehrende größere Textbausteine hin durch, zeigt sich, dass es fünf Kernthemen gibt, denen sehr viele Ratgeber Teile, Kapitel oder Teilkapitel widmen: 
(1) Angst an sich: die entsprechenden Teile, Kapitel oder Teilkapitel tragen Titel wie „WAS IST ANGST?“ (Priest 1983/1985: 8), „Zum Verständnis von Angst“ (Schmidt-Traub 1995: 5) oder „Angst als natürliche Reaktion auf Gefahr“ (Craske \& Barlow 2006/2016: 37);

(2) Arten von Angst bzw. ,Angststörungen': die Abschnitte zu diesem Thema heißen etwa „Spielarten der Angst“ (Lückert 1993: 36), „Die verschiedenen Formen der Angst“ (Peiffer 2004: 42) oder „Welche Formen der Angststörung gibt es?““(Voos 2015: 25);

(3) Ursachen von Angst bzw. ,Angststörungen': die Abschnitte, die dieses Thema behandeln, sind z. B. mit „Warum haben wir Angst?“ und „Wie entstehen Angststörungen?“ (Wittchen 1997/1999: 11, 43), „Woher kommt die Angst und was lässt sie wachsen?“ (Wilms \& Wilms 2008: 53) oder „Entstehung einer Angsterkrankung“ (Illy 2016: 25) betitelt;

(4) Maßnahmen gegen Angst, die die/der Lesende allein ergreifen kann: die entsprechenden Teile, Kapitel oder Teilkapitel haben Überschriften wie „SELBSTHILFEWEGE ZUR BEWÄLTIGUNG VON ANGST UND DEPRESSIONEN“ (Priest 1983/1985: 26), „Die Symptome in den Griff bekommen“, „Negative Denkmuster überwinden“, „Systematische Desensibilisierung“ usw. (Peurifoy 1997/2007: 85, 99, 113) oder „TRUST - and go! Das KRISEN-ABC“ (Diegelmann \& Isermann 2011/2012: 46);

(5) Maßnahmen gegen Angst, an denen eine andere Person mitwirken muss: Bezeichnungen für Abschnitte $\mathrm{zu}$ diesem Thema sind beispielweise „Die ärztliche Behandlung“ (Trickett 1992/1995: 33), „Was Therapeuten tun können“ (Wilms \& Wilms 2008: 79) oder „Medikamente gegen die Angst“ (Craske \& Barlow 2006/2016: 153).

Die Zitate zeigen, dass die Überschriften bzw. Titel für die entsprechenden Abschnitte häufig aus Fragen bestehen, auf die der folgende Text Antworten bietet, und aus Nominalphrasen, die das Thema bezeichnen. Die Zitate lassen zudem erkennen, dass ein Kernthema auch unter mehreren Überschriften bzw. Titeln abgehandelt werden kann. Wie bereits angedeutet, enthalten nicht alle Ratgeber Teile, Kapitel oder Teilkapitel zu allen Kernthemen, und natürlich finden sich in vielen Ratgebern Teile, Kapitel oder Teilkapitel zu weiteren Themen. Die genannten Kernthemen machen aber normalerweise einen Großteil der Gesamttexte aus und werden in der angegebenen Reihenfolge abgehandelt. 
Hinsichtlich des Aufbaus und der Themen sind die analysierten Ratgeber also relativ musterhaft. Ein diachroner Wandel zeichnet sich diesbezüglich nicht ab.

Die Liste der Kernthemen lässt Rückschlüsse darauf zu, wie die Ratgeber das Thema Angst bearbeiten: Auf einen definitorisch-begriffsbestimmenden Zugang folgt üblicherweise eine differenzierend-klassifizierende, explizierendbegrün-dende und direktiv-instruierende Entfaltung des Themas. Die untersuchten Ratgeber gegen Angst ,zeichnen sich“ folglich „durch die Grundfunktionen der Information und der Appellation aus“, die in der Forschungsliteratur für Ratgeber generell angenommen werden (Niemann 2018: 77). Die Annahme schließt freilich nicht aus, dass das Verhältnis von Information und Appellation über verschiedene Ratgeber bzw. Typen von Ratgebern hinweg variiert. Wenn die Wissensvermittlung relativ stark akzentuiert wird, wie es in den untersuchten Angst-Ratgebern der Fall zu sein scheint, ist das - kulturanalytisch betrachtet - möglicherweise damit zu erklären, dass Wissen als Voraussetzung oder zumindest als begünstigender Faktor für eine veränderte Praxis aufgefasst wird, für die Ratgeber ja gemäß der obigen Bedeutungsbeschreibung Anleitungen oder Tipps geben. Eine ausgeprägte Wissensvermittlung kann zugleich die öffentliche Wahrnehmung des jeweiligen Gebiets - in diesem Falle des Umgangs mit Angst - beeinflussen. Sie kann z. B. dessen Wahrnehmung als professionalisierten Wissens- oder sogar Wissenschaftsbereich verstärken, in dem ein deutliches Kompetenzgefälle zwischen ,Experten` und ,Laien` besteht.

\subsection{Bestimmungen von Angst}

Die Auswertung des jeweils ersten Kapitels oder Teilkapitels, das Angst bestimmt, ergibt, dass die Angst-Ratgeber darin hochgradig musterhaft verfahren. Eine besonders wichtige Rolle spielt die Unterordnung des Angstbegriffs unter einen Oberbegriff. Kaum überraschend wird Angst am häufigsten als ein Gefühl oder eine Reaktion bestimmt, z. B. so:

(1) „Jeder kennt das Gefühl von Angst“ (Schmidt-Traub 1995: 5),

(2) „Sie [Angst] ist ein archaisches, tief in der tierischen und menschlichen Natur verwurzeltes Reaktionsmuster“ (Peseschkian \& Boessmann 1998: 12),

(3) „Angst ist ein grundlegendes und normales Gefühl“ (Wittchen 1997/1999: 6), 
(4) „wir alle kennen Angstgefühle aus verschiedenen Lebenssituationen, in die wir ab und zu geraten“" (Peiffer 2004: 35),

(5) „Angst ist zuallererst ein lebenswichtiges Gefühl“ (Wilms \& Wilms 2008: 13),

(6) „Angst ist eine natürliche Schutzreaktion, ähnlich wie Schmerz“ (Voos 2015: 13),

(7) „Angst ist eine ganz normale Reaktion des Körpers und zählt gewissermaßen zu unserer emotionalen Grundausstattung, ebenso wie Freude“ (Illy 2016: 5).

Dass Angst eng mit Gefühl verbunden ist, zeigt sich auch in den Kollokationen $\mathrm{zu}$ Angst in den drei digitalen Korpora. ${ }^{3}$ Sieht man die Kollokationen der Teilkorpora auf Ausdrücke durch, die Oberbegriffe von Angst bezeichnen könnten, findet man darunter stets Flexionsformen des Lexems Gefühl (vgl. Tabelle 1).

Tab. 1: Ausdrücke für mögliche Oberbegriffe in den Kollokationen zu Angst in den drei digitalen Teilkorpora

\begin{tabular}{lll}
\hline $\begin{array}{l}\text { Ratgeber aus den } \\
\text { 1980er und 1990er Jahren }\end{array}$ & Ratgeber aus den & Ratgeber aus den \\
& 2000er Jahren & 2010er Jahren \\
\hline gefühl & erleben & emotion \\
& gefühle & erkrankungen \\
& gefühl & erleben \\
& gedanken & gefühl \\
& & gefühle \\
& gedanken \\
\hline
\end{tabular}

In den ausgewerteten Abschnitten der Ratgeber wird eine differentia specifica sehr viel seltener genannt als ein Oberbegriff oder genus proximum. Vergleichbares gilt für die Nennung von Synonymen und eindeutig erkennbaren Paraphrasen. Unter Rückgriff auf die Konzepte, die Reinhard Fiehler für die „Thema-

3 Alle Kollokationen, die in diesem Teil erwähnt werden, sind für Angst als Lemma berechnet worden (span: 5<>5, statistic: LogRatio, statistic value threshold: 3.0 , collocation frequency threshold: 10). Den korpuslinguistischen Analysen liegen stets alle digitalisierten Teile der Ratgeber zugrunde. 
tisierung von Erleben und Emotionen“ einführt, könnte man sagen, dass sowohl von alternativen „begrifflichen Erlebens- und Emotionsbenennungen“ als auch von „Erlebens- und Emotionsbeschreibungen“ relativ wenig Gebrauch gemacht wird (Fiehler 2001: 1431, vgl. 1431-1432).

Wiederholt wird allerdings gesagt oder zumindest impliziert, dass Angst in Zusammenhang mit der Wahrnehmung einer tatsächlichen oder vermeintlichen Bedrohung oder Gefahr stehe bzw. mit der begründeten oder unbegründeten Antizipation von nachteiligen Ereignissen zu tun habe. So heißt es beispielsweise (freilich mit einigen sprachlichen und logischen Ungereimtheiten, wie sie in den Ratgebern ab und zu vorkommen):

(1) „Angst ist das Gefühl, zu glauben, daß bald etwas Unangenehmes passieren wird“ (Priest 1983/1985: 8),

(2) „Bei allen höheren Lebensformen löst eine lebensbedrohliche Si-tuation [sic] einen emotionalen Stresszustand aus. Während Tiere in einer solchen Gefahrensituation eben mit angeborenen Verhal-tensweisen [sic] reagieren, ruft eine gleichartige Bedrohung beim Men-schen [sic] zunächst Angst hervor“ (Hoffmann 2007: 30),

(3) „Angst tritt immer dann auf, wenn wir uns bedroht fühlen, sei es durch einen drohenden Unfall oder einen unheimlichen Film im Kino“ (Illy 2016: 5).

Eine Abgrenzung von Angst gegenüber Furcht ist hingegen nur in Ausnahmefällen $\mathrm{zu}$ beobachten, obwohl für diese zwei Lexeme zurückgehend auf Sören Kierkegaard (vgl. 1844/2005: 41-46) und Martin Heidegger (vgl. 1927/1977: 140142, 184-191) immer wieder eine „semantische Divergenz postuliert wird: Furcht sei ein gegenstandsgerichtetes, Angst hingegen ein gegenstandsloses, frei flottierendes Gefühl“ (Filatkina 2016: 67, Hervorheb. i. O., vgl. 67-68 sowie ausführlicher zu dieser Tradition Bergenholtz 1980: 66-68). Die Bestimmungen von Angst wie auch der sonstige Sprachgebrauch in den Ratgebern entsprechen dieser Unterscheidung meist nicht, was offenbar in ähnlicher Weise auf den alläglichen Sprachgebrauch außerhalb der Ratgeber zutrifft (vgl. Bibliographisches Institut 2019a).

Sehr verbreitet ist die Erwähnung von positiven oder negativen Folgen von Angst, die in der Regel mit einer bestimmten Stärke von Angst assoziiert werden. Wie es sich in den obigen Zitaten schon angedeutet hat, wird moderate und/oder begründete Angst oft als normal, natürlich und nützlich beschrieben; 
es wird behauptet, dass sie positive Folgen habe. Angst, die als unverhältnismäßig stark bzw. nicht ausreichend unbegründet beurteilt wird, gilt jedoch als dysfunktionales Phänomen mit negativen Folgen:

(1) „Angst ist in wirklich brenzligen Situationen außerordentlich nützlich, denn sie wirkt wie ein Warnsystem und macht uns vorsichtig. [...] Angst ist somit lebenswichtig. Es wäre demnach unvernünftig und sogar gefährlich, ein Leben ohne Angst anzustreben. Sind demgegenüber Angststörungen, wie Panik und Agoraphobie, unsinnig, qualvoll und einengend? Richtig!“ (Schmidt-Traub 1995: 7-8),

(2) „Kleine Angstspannungen (,Lampenfieber`) steigern [...] die seelische und körperliche Leistungsfähigkeit; heftige Angst hingegen blockiert die geistige Konzentration, lähmt die Bewegungen und engt die Wahrnehmung ein. Während die normale Angst vor einer wichtigen Situation, deren Gelingen intensiv gewünscht wird, im Erleben der ersten erfolgreichen Schritte verschwindet, bleibt die neurotische Angst bestehen und lähmt die geistige Leistung“ (Schmidbauer 2005: 16),

(3) „Angst kann außerordentliche Kräfte verleihen. Sie kann ungeahnte Kreativität fördern. Sie kann helfen, eine Aufgabe klarer ins Visier zu nehmen und besser zu bewältigen, als wir es uns zutrauen. Angst kann uns Flügel verleihen, mit denen wir uns aufschwingen zu sensationellen Erfolgen. Aber sie kann uns auch lähmen, zu Entscheidungsunfähigkeit verdammen, unseren Körper auszehren, unsere geistige Leistung verringern, uns den Spaß am Leben nehmen“ (Schmitz \& Schmitz 2005: 23),

(4) „Die Angst schützt uns vor waghalsigen Aktionen und lässt uns die Dinge überdenken, bevor wir sie tun“(Voos 2015: 13),

(5) „Bei Angsterkrankungen hingegen können Ängste die psychische Gesundheit dauerhaft beeinflussen. Sie können den Alltag beispielsweise dadurch beeinträchtigen, dass sie auch dann auftreten, wenn gar keine offensichtliche Gefahr droht. [...] Die Angst hat sich im Rahmen der Erkrankung also auch auf eigentlich angstfreie Lebensbereiche ausgedehnt. [...] Die natürlichen Ängste hingegen, die soll bitte jeder behalten, sie sind überlebenswichtig“ (Illy 2016: 7). 
Aus den Beispielen geht auch hervor, dass positiv beurteilter Angst öfters eine Leistungssteigerungs- und (teilweise als Folge davon) eine Warn- oder Schutzfunktion zugesprochen wird. In den entsprechenden Textstellen oder in ihrem Kotext finden sich mehrfach Metaphern für oder Vergleiche mit Angst bzw. Angsteffekte/n, die den Ausdruck Alarm enthalten, z. B. „Alarmreaktion“ (Schmidt-Traub 1995: 7), „Alarmsystem“ (Schmitz \& Schmitz 2005: 22), „Alarmanlage“ (Hoffmann 2007: 9) oder „Alarmfunktion“ (Illy 2016: 5). Solche Metaphern und Vergleiche unterstreichen die warnende und schützende Funktion von Angst, lassen sie aber auch als erschreckend und aufrüttelnd erscheinen. Negativ beurteilte Angst wird im Kontrast dazu häufiger als etwas dargestellt, das das Wohlbefinden und/oder die Leistung reduziere. Diese und andere nachteilige Folgen werden mehrfach mit Metaphern der Raumbegrenzung und Bewegungseinschränkung formuliert, so in den obigen Beispielen etwa mit einengend, blockiert, lähmt, engt ... ein, lähmen. Dies weist Angst einen hinderlichen, behindernden Charakter zu und entspricht zugleich der Etymologie von Angst: Das Wort geht auf indogermanisch „„ang ${ }^{\text {h }} \mathrm{u}-$,eng, bedrängend““ zurück (Kluge \& Seebold 1883/2012, Hervorheb. i. O.).

Dafür, dass Angst als graduelles Phänomen konzeptualisiert wird, lassen sich auch in den Kollokationen zu Angst Anhaltspunkte finden. In den Kollokationslisten der Teilkorpora für alle drei Zeitphasen finden sich verschiedene Gradausdrücke, also Ausdrücke, die ein Mehr oder Weniger bezeichnen können (vgl. Tabelle 2). Die Gradausdrücke nehmen im Laufe der Zeit deutlich zu.

Tab. 2: Gradausdrücke in den Kollokationen zu Angst in den drei digitalen Teilkorpora

\begin{tabular}{llll}
\hline $\begin{array}{l}\text { Ratgeber aus den } \\
\text { 1980er und 1990er Jahren }\end{array}$ & $\begin{array}{l}\text { Ratgeber aus den } \\
\text { 2000er Jahren }\end{array}$ & $\begin{array}{l}\text { Ratgeber aus den } \\
\text { 2010er Jahren }\end{array}$ & \\
\hline starke & verlieren & maximale & ausmaß \\
meisten & stärker & stärke & stark \\
besonders & große & übermäßigen & verlieren \\
etwas & weniger & normale & weniger \\
mehr & meisten & starken & mehr \\
nur & leicht & große & nur \\
sehr & sehr & verstärkt & sehr \\
& nur & reduzieren & \\
& viel & verstärken & \\
& besonders & starke & \\
\hline
\end{tabular}


Im jeweils ersten Kapitel oder Teilkapitel der Ratgeber, in dem Angst bestimmt wird, fällt schließlich noch eine weitere Regelmäßigkeit auf: Immer wieder wird konstatiert, dass Angst neben einer mentalen auch eine körperliche Dimension habe, was zur Etymologie des Wortes passt. Manchmal wird zusätzlich eine das Verhalten betreffende Dimension erwähnt. Allerdings schwanken die Bezeichnungen für das, was ich hier faute de mieux Dimensionen genannt habe; nach den Bezeichnungen zu urteilen, werden die Dimensionen zum Teil als Ausprägungen oder Elemente von Angst verstanden, zum Teil aber auch als Begleiterscheinungen von oder Reaktionen auf Angst:

(1) „Bis jetzt habe ich davon gesprochen, was in Ihrem Kopf vorgeht, wenn Sie Angst haben. Die meisten Gefühle haben aber auch physische Auswirkungen, und Angst ist keine Ausnahme“ (Priest 1983/1985: 10),

(2) „Ganz gleich, ob eine echte Bedrohung vorliegt oder bloß eine Gefahr auf der Vorstellungsebene, das daraufhin entstehende Angstgefühl setzt sich aus 3 Reaktionsebenen zusammen:

1) Körperliche und physiologische Beschwerden [...]

2) Angst hat auch eine verbal-kognitive Seite. [...]

3) Das Angstgefühl hat noch eine motorische und Verhaltensebene“ (Schmidt-Traub 1995: 12, 14-15, Hervorheb. i. O.),

(3) „Angst in Form von körperlichen Veränderungen, ängstlichen Gedanken und Gefühlen sowie ängstliches Verhalten tritt zumeist in Situationen auf, die wir als bedrohlich, ungewiß und unkontrollierbar empfinden“ (Wittchen 1997/1999: 6),

(4) „Wissenschaftler teilen Angst oft in drei Komponenten ein: die körperliche Komponente, die kognitive (Gedankens-) [sic] Komponente und die Verhaltenskomponente“ (Craske \& Barlow 2006/2016: 41).

Auch in den Kollokationen zu Angst zeichnen sich Spuren der drei genannten Dimensionen ab. Die Kollokationen aller Teilkorpora enthalten Ausdrücke zu deren Bezeichnung (vgl. Tabelle 3). 
Tab. 3: Mögliche Ausdrücke für die drei Dimensionen von Angst in den Kollokationen zu Angst in den drei digitalen Teilkorpora (ohne Flexionsformen der Lexeme Emotion, Gefühl und Gedanke; für diese vgl. Tabelle 1)

\begin{tabular}{lll}
\hline Ratgeber aus den & Ratgeber aus den & Ratgeber aus den \\
1980er und 1990er Jahren & 2000er Jahren & 2010er Jahren \\
\hline körperlichen & körperlichen & vermeiden \\
körperliche & körperliche & körperlichen \\
denken & fühlen & vermeidungsverhalten \\
& denken & umgang \\
& & körperliche \\
\hline
\end{tabular}

Die vorgestellten Ergebnisse belegen, dass die Bestimmungen von Angst in den analysierten Ratgebern über die Dekaden weitgehend konstant bleiben. Zwar variieren die Bestimmungen von Ratgeber zu Ratgeber natürlich in Details, entscheidende Elemente sind aber bei vielen Ratgebern sehr ähnlich, und bei diesen lässt sich keine eindeutige Veränderung identifizieren.

Ein weiterer Aspekt fällt bei der Reflexion der Ergebnisse auf: Angst wird zwar immer wieder als Gefühl oder Emotion bestimmt, was diese/s aber eigentlich ausmacht, d. h. wie sich Angst wirklich anfühlt, bleibt merkwürdig unbestimmt. Daran ändert auch die Erwähnung dreier Dimensionen von Angst - der mentalen, körperlichen und das Verhalten betreffenden - nichts: denn wenn diese Dimensionen als Begleiterscheinungen von oder Reaktionen auf Angst begriffen werden, handelt es sich um Phänomene, die mit Angst nicht identisch sind; und wenn die Dimensionen alternativ als Ausprägungen oder Elemente von Angst aufgefasst werden, steht das im Widerspruch zur Bestimmung von Angst als Gefühl, zumindest sofern man dem alltagssprachlichen Verständnis von Gefühl folgt. Sogar der Duden ist in seiner Bedeutungsbeschreibung von Angst präziser als die meisten Ratgeber: „mit Beklemmung, Bedrückung, Erregung einhergehender Gefühlszustand [angesichts einer Gefahr]; undeutliches Gefühl des Bedrohtseins“ heißt es dort (Bibliographisches Institut 2019a). Erst recht gilt das für psychologische Nachschlagewerke wie etwa das bekannte „Dorsch. Lexikon der Psychologie“, in dem Angst u. a. als „emot. Zustand [...], gekennzeichnet durch Anspannung, Besorgtheit, Nervosität, innere Unruhe und Furcht vor zukünftigen Ereignissen" erläutert wird (Asendorpf \& Caspar 2019). Erklären ließe sich die Unterbestimmung von Angst in den Ratgebern mit dem hochgradig subjektiven Charakter, den Angst mit allen anderen Emotionen teilt. Ein weiterer Grund könnte sein, dass das Sprechen und Schreiben über Angst in der Alltagssprache immer noch relativ tabuisiert ist und deshalb verhältnismäßig wenig Routinen zur Formulierung der Qualitäten des Angstge- 
fühls bereitstehen. Allerdings gäbe es auch für die Verbalisierung von schwer Verbalisierbarem etablierte Verfahren, etwa den Rückgriff auf ungewöhnliche Metaphern oder Vergleiche (zu Vergleichen und weiteren Verfahren, mit denen extreme Angst in Gesprächen kommuniziert wird, vgl. Günthner 2006: 126-146; zu Kombinationen von Verfahren, mit denen verschiedene Arten von Angst in Gesprächen kommuniziert werden, vgl. Lindemann 2012: 139-174). Zudem ist der Angstwortschatz im gegenwärtigen Deutschen offenbar durchaus differenziert - Christopher Georgi (2018: 123) beispielsweise kommt jüngst auf ,insgesamt 162 Wörter, die auf 46 Nomen, 17 Verben und 99 Adjektive zurückzuführen sind“ und von „Entsetzen“, „Schrecken“, „Panik“ oder „Horror“ über „traumatisierend“, „bedrohlich“ oder „unheimlich“ bis hin zu „graulen“, „fürchten“, „grauen“ oder „Bange Machen“ reichen (vgl. außerdem den umfangreichen Eintrag zu „Furcht, Schrecken“ in Dornseiff \& Quasthoff 1934/2004: 188 sowie zu den zahlreichen Phraseologismen im Deutschen, die sich vorrangig auf Angst beziehen, Cieślarová 2012: 8-14). Dass die Ratgeber Angst vergleichsweise unbestimmt lassen, ist also kaum darin begründet, dass sie nicht anders können. Vermutlich sehen ihre Autoren entweder keine Notwendigkeit für eine genauere Bestimmung, oder sie unterlassen diese aus verkaufsstrategischen Gründen: Je weniger präzise sie ihren zentralen Gegenstand umreißen, desto größer ist das Publikum, das sich von ihrem Buch angesprochen fühlen kann. Diese Offenheit des Angstbegriffs kann allerdings bei der Selbsttherapie von Angst mithilfe von Ratgebern problematische Konsequenzen haben, wenn die Autoren und die Leserschaft unter Angst nicht dasselbe verstehen.

Kulturanalytisch interessant ist darüber hinaus das Oszillieren der Ratgeber zwischen der Normalisierung und Pathologisierung von Angst: Angst wird einerseits als üblich und sinnvoll dargestellt, wodurch sich Lesende, die Angst haben, im Bereich des ,Normalen“ verorten können. Andererseits wird zu viel Angst als problematisch präsentiert, wodurch sich Lesende mit Angst auch behandlungsbedürftig fühlen können. Dass die Ratgeber die Grenze zwischen ,normaler` Angst und zu viel Angst u. a. mithilfe des Leistungsbegriffs ziehen, macht es für die/den einzelne/n Lesende/n wohl nicht einfacher, die Grenze zwischen beidem zu finden. Das Vorgehen ist aber insofern signifikant, als es Leistung und Leistungsfähigkeit als wichtige kulturspezifische Werte sowohl ausdrückt als auch bestätigt und verstärkt.

Wie erwähnt, schreiben die Ratgeber regelmäßig, dass Angst mit der begründeten oder unbegründeten Antizipation von nachteiligen Ereignissen zu tun habe. Das legt die Frage nahe, was vielen von ihnen als solch ein nachteiliges Ereignis gilt, d. h. die Frage danach, wovor man ihnen zufolge Angst haben kann. Um diese Frage zu beantworten, habe ich in den Ratgeberkorpora aller 
drei Zeitphasen nach dem Ausdruck Angst vor gesucht. Angst vor kommt insgesamt $470 \mathrm{Mal}$ vor - während Angst um interessanterweise lediglich neun Mal gebraucht wird. Sämtliche KWICS des Ausdrucks Angst vor habe ich nach der Semantik der Substantive klassifiziert, die von der Präposition regiert werden und Angstauslöser bezeichnen. Für alle drei Zeitphasen lassen sich sechs Klassen von Substantiven bilden:

Die Ratgeber thematisieren immer wieder Angst vor Tieren (z. B. vor Hunden, Mäusen, Schlangen, Spinnen), vor Menschen oder Menschengruppen (z. B. vor dem Chef, dem Zahnarzt, vor Fremden), vor Reaktionen von oder Relationen $\mathrm{zu}$ anderen Menschen (z. B. vor einem Beziehungsverlust, einem Konflikt, vor Kritik, Nähe, Vereinsamung), vor dem Tod, dem Sterben oder, seltener, dem Leben generell, vor anderen äußerlich erkennbaren Situationen oder Aktivitäten (z. B. vor Auftritten, vor dem Aufzugfahren, dem Autofahren, vor Enge, vor dem Fliegen, vor Höhen, Kontrollverlust, Krankheit, Plätzen, Schmutz, Versagen) und vor Gefühlen oder Gedanken (z. B. vor Angst, vor einer Panikattacke, vor Schmerzen). Nur ganz wenige Substantive lassen sich diesen Klassen nicht zuordnen. Abbildung 1 und 2 zeigen, wie sich die Substantive in den drei Teilkorpora auf die verschiedenen Klassen verteilen.

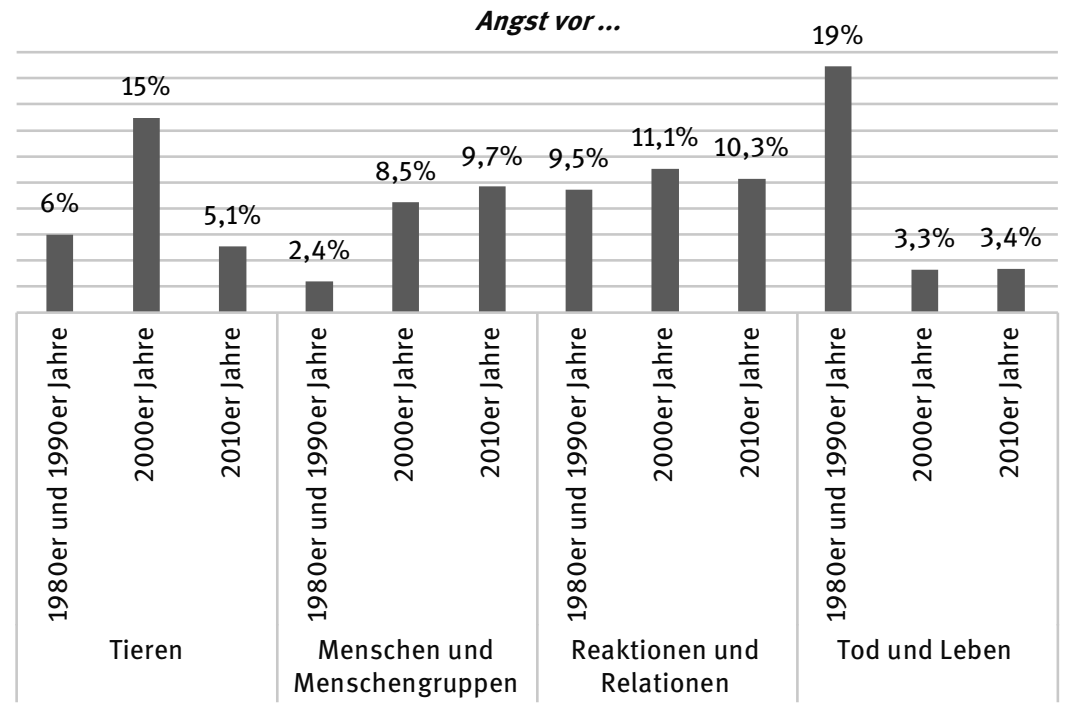

Abb. 1: Verteilung der Substantive, die von der Präposition regiert werden und Angstauslöser bezeichnen, auf semantische Klassen (Gesamtzahl der Klassifikationen im digitalen Teilkorpus der 1980er und 1990er Jahre: 166; der 2000er Jahre: 169; der 2010er Jahre: 178) 
Angst vor...

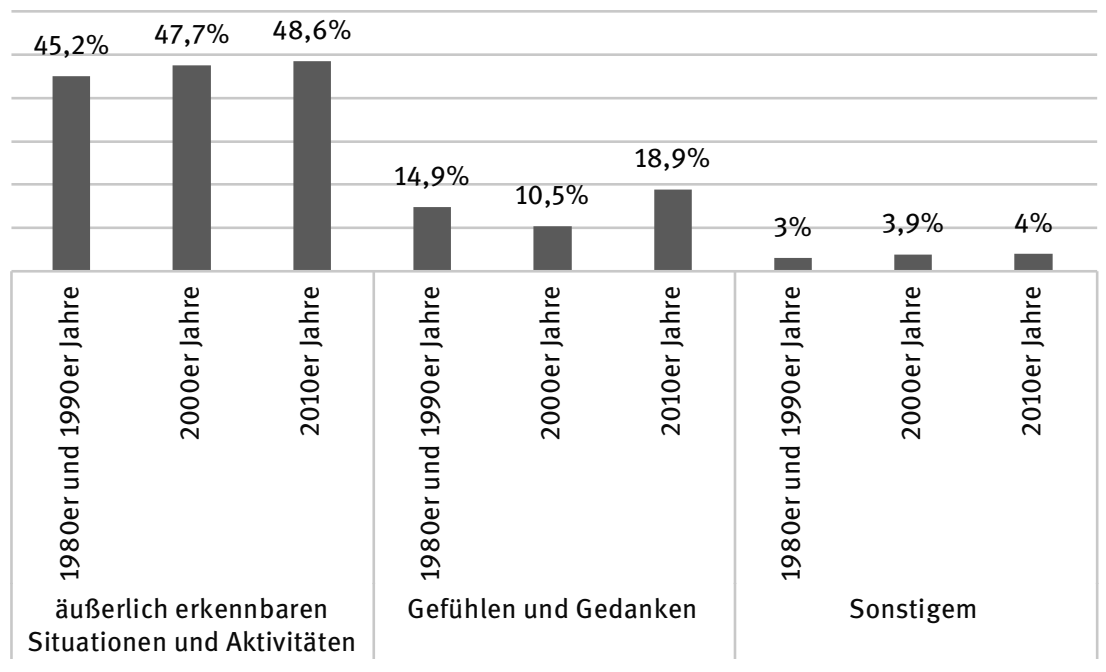

Abb. 2: Verteilung der Substantive, die von der Präposition regiert werden und Angstauslöser bezeichnen, auf semantische Klassen (Gesamtzahl der Klassifikationen im digitalen Teilkorpus der 1980er und 1990er Jahre: 166; der 2000er Jahre: 169; der 2010er Jahre: 178)

Auffällig ist die zirkuläre Entwicklung der Erwähnung von Tieren, die Abnahme der Erwähnung von Tod und Leben und die Zunahme der Erwähnung von Menschen und Menschengruppen sowie - der Tendenz nach - auch von Gefühlen und Gedanken. Möglicherweise ist diese Verschiebung zumindest indirekt mit einer länger andauernden historischen Entwicklung verbunden, durch die laut Käuser (2013: 142) „Angst [als für die Moderne seit 1800 typisches Phänomen] nicht mehr von Naturobjekten [...] bestimmt, sondern autoreferentiell und medienkulturell vom Menschen hervorgebracht [ist]“.

Die zahlreichen Vorkommen von Angst vor und die konstante Vervollständigung der Phrase mit Substantiven aus denselben sechs semantischen Klassen deuten in jedem Fall darauf hin, dass es eine Art ,Kanon' von Angstauslösern gibt. Die einbezogenen Ratgeber scheinen davon auszugehen, dass es ein Set von Entitäten gibt, auf die sich Angst, und zwar gerade solche, die als unverhältnismäßig bzw. unbegründet beurteilt wird, typischerweise bezieht. Viele der Angstauslöser, die in den Ratgebern öfters angesprochen werden, überraschen wenig, z. B. Beziehungsverlust, Krankheit oder Tod. Erstaunlicher ist, dass viele große zeitgenössische Herausforderungen und Probleme kaum Erwähnung finden: Nur selten wird etwa Angst vor sozialem Abstieg und Arbeitslosig- 
keit (drei Mal), vor Terror(anschlägen) (drei Mal) oder vor Auswirkungen der Umweltzerstörung und des Klimawandels (zwei Mal) genannt. Dazu stehen repräsentative Umfragen im Kontrast, nach denen ein großer Anteil der Wohnbevölkerung Deutschlands ab 14 Jahren Angst vor diesen und ähnlichen Entwicklungen bzw. Ereignissen hat (vgl. Infocenter der R+V-Versicherung 2019). Die festgestellten sprachlichen Muster könnten dadurch verursacht sein, dass die meisten Menschen, die wegen besonders ausgeprägter oder als unbegründet empfundener Angst Rat oder Hilfe suchen, tatsächlich Angst vor den genannten Entitäten haben, wofür sowohl anthropologisch-universelle als auch kulturelle Ursachen infrage kommen (zu den Konjunkturen verschiedener Ängste im 20. Jahrhundert, die auf die kulturelle Prägung von Angstauslösern hinweisen, vgl. Bourke 2005/2006). Denkbar ist aber ebenso, dass besonders über die genannten Angstauslöser geschrieben wird, weil diese gesellschaftlich relativ wenig tabuisiert und deshalb am häufigsten kolportiert worden sind. Ein wichtiger Effekt davon, dass die Ratgeber bevorzugt über Angst vor ganz bestimmten Entitäten schreiben, könnte darin bestehen, dass Angst genau davor als immerhin relativ normal gilt, selbst wenn sie als unverhältnismäßig bzw. unbegründet beurteilt wird. Anders formuliert: Wenn jemand Angst vor einer der vielgenannten Entitäten hat und diese Angst als irrational oder beeinträchtigend empfindet, kann sie/er sich nach der Lektüre von Angst-Ratgebern zumindest damit beruhigen, sich mit dieser Angst in zahlreicher Gesellschaft zu befinden. Im Gegenzug wird Angst, die sich auf eine vollkommen andere Größe richtet, von den Menschen, die sie erleben, vermutlich als besonders seltsam und besorgniserregend eingeschätzt.

\subsection{Formulierung von Zielen}

Da Ratgeber, wie oben beschrieben, dadurch gekennzeichnet sind, dass sie Anleitungen, Tipps o. Ä. für die Praxis auf einem bestimmten Gebiet geben, haben sie stets auch ein Ziel oder eine Idealnorm, der die Lesenden mit den gegebenen Anleitungen, Tipps bzw. Ratschlägen näherkommen sollen. Analysiert man das Vorwort, die Einleitung oder einen vergleichbaren Abschnitt zu Beginn der ausgewählten Angst-Ratgeber mit Blick auf Muster in den Zielformulierungen, wird zunächst deutlich, dass Ziele für mehrere Zielgruppen genannt werden: für Menschen, die Angst haben, oft aber auch für deren Bezugspersonen, seltener für Menschen, die bislang nur wenig Angst haben, und für therapeutisches Fachpersonal. Im Folgenden konzentriere ich mich auf die primäre Zielgruppe der Ratgeber, d. h. auf Personen, die Angst haben, und auf die Ziele, die für sie erwähnt werden. 
Die Zielgruppe der Personen, die Angst haben, wird in den einführenden Kapiteln unterschiedlich bezeichnet: Am häufigsten sind Bezeichnungen mit betroffene/Betroffene, Menschen, die/Menschen mit und Anreden mit dem Pronomen Sie und seinen Flexionsformen. Es heißt z. B.:

(1) „Mit diesem Buch verfolge ich nicht die Absicht, Ihnen die Inanspruchnahme einer Psychotherapie zu empfehlen“ (Lückert 1993: 8),

(2) „Ich würde mich sehr freuen, wenn dieses Buch viele Betroffene [...] erreicht“(Schmidt-Traub 1995: VI),

(3) „Mit ihm [diesem Buch] möchte ich allen Betroffenen einen praktischen und hilfreichen Wegweiser an die Hand geben“ (Trickett 1992/1995: 11),

(4) „Mein Ziel war es, über mein erstes Buch hinauszugehen, neue Gebiete zu erschließen und Menschen, die unter Angstproblemen leiden, einen Weg $\mathrm{zu}$ weisen, der sie $\mathrm{zu}$ einer langfristigen Genesung führt“ (Peurifoy 1997/2007: 9),

(5) „Dies ist ein Selbsthilfebuch für Menschen mit psychischen Problemen oder gar Erkrankungen wie Depressionen, Burnout oder Angststörungen“ (Hansch 2011: 2).

Pronomen der dritten Person Plural werden in den ausgewählten Ratgebern konstant zur Bezeichnung der Hauptzielgruppe gebraucht. Bezeichnungen mit betroffene/Betroffene nehmen indes im Laufe der Zeit ab, während solche mit Menschen, die/Menschen mit zunehmen. Dieser Wandel könnte mit der latent abwertenden Semantik zu tun haben, die betroffen bzw. Betroffene gegenwärtig hat. Das DWDS-Wortprofil von Betroffene weist deutlich darauf hin, dass Betroffene, salopp gesagt, ein Problem haben: Unter den Top-20-Kollokationen zu Betroffene finden sich „klagen“, „wehren“, „entschädigen“, „helfen“, „Leid“ und „leiden“ (Digitales Wörterbuch der deutschen Sprache 2019).

Zeitübergreifend zielen die ausgewählten Angst-Ratgeber nach ihren eigenen Angaben darauf ab, dass Menschen mit Angst diese als weniger problematisch empfinden. Das jeweilige Buch soll dabei wahlweise der Notwendigkeit einer Psychotherapie vorbeugen, diese ersetzen, vorbereiten oder begleiten. Das übergreifende Ziel wird sprachlich unterschiedlich konturiert, wobei eine diachrone Veränderung erkennbar ist. Mit Ausdrücken wie bewältig-, fertig werden, -heil-, -leid- u. a. bezeichnet eine Reihe älterer Ratgeber das Ziel, Angst abzu- 
schwächen, gar aufzulösen oder, allgemeiner formuliert, das, was als Defizit bzw. als negativ gilt, abzubauen:

(1) „Vielleicht hilft es [dieses Buch] auch Ihnen, Ihre Angstzustände zu bewältigen. [...] Ich wünsche Ihnen gutes Vorankommen bei der Bewältigung Ihrer Angst“"(Schmidt-Traub 1995: VI),

(2) „Ich versichere Ihnen, daß Panikattacken heilbar sind. Ja, heilbar. Natürlich erfolgt die Heilung nicht einfach durch die Lektüre der folgenden Kapitel und auch nicht über Nacht. Aber ich hoffe, daß sich Ihre Angst verringert, sobald Sie verstehen, was mit und in Ihnen während solcher Attacken vor sich geht“ (Trickett 1992/1995: 11),

(3) „Finden Sie sich also nicht mit Ihrer Angst ab, sondern arbeiten Sie die Techniken in diesem Band durch. Ängste kann man loswerden. [...] Fangen Sie also gleich heute damit an, Ihrer Angst Kontra zu geben!“ (Peiffer 2004: 7).

Im Gegensatz dazu formulieren mehrere der neueren Ratgeber mit Ausdrücken wie umgehen/Umgang, aktivier-, kraft-/kräft-, ressource-, -freude- usw. das Ziel, $\mathrm{zu}$ einer neuen Haltung gegenüber Angst und zu einer veränderten Handhabung dieser zu gelangen, Gegenkräfte zu Angst zu fördern, und generell das, was als (potenzielle) Stärke bzw. als positiv betrachtet wird, auszubauen. Parallel dazu wird die angenommene Ausgangssituation - Angst, die als problematisch empfunden wird - zunehmend mit den Ausdrücken (Angst)Störung und (Angst)Erkrankung bezeichnet und damit eindeutig als Krankheit kategorisiert:

(1) „Grundsätzlich gibt es ja zwei Wege, psychische Störungen zu bessern: Zum einen kann man versuchen, den Defekt zu reparieren. Oft verschwinden Defekte aber auch per Selbstheilungskraft, wenn man - und das ist der zweite Weg - die positiven und gesunden Anteile eines Menschen stärkt und entwickelt. Beide Wege sind wichtig, der zweite aber ist zumeist der wichtigere und bessere. Und für einen gesunden Umgang mit sich selbst, für den Aufbau eines gelingenden und erfüllten Lebens gibt es ein Grundwissen und Grundprinzipien, die einen hohen Grad an Allgemeingültigkeit aufweisen. Dieses Basiswissen zur Selbststeuerung und Selbstentwicklung, das wir alle schon in der Schule hätten lernen müssen, wird Ihnen im vorliegenden Buch vermittelt“ (Hansch 2011: 3), 
(2) „Dieses Buch stellt konkrete Schritte zur Stärkung der eigenen Kompetenzen und zur Erweiterung des eigenen Handlungsspielraums im Angesicht von Angst, Krisen und unerwarteten Schicksalsschlägen zur Verfügung. [...] Im Mittelpunkt stehen [...] die Stärkung und Erweiterung individueller Ressourcen (Kraftquellen) auch angesichts existenzieller Grenzerfahrungen. Ressourcen können aber auch prophylaktisch aktiviert werden, damit bisher noch nicht entfaltete oder vergrabene Potenziale spürbar werden. [...] Wir wollen Mut machen, mittels konkreter Erfahrungen neue innere und äußere Wege aus diesem Ohnmachtsgefühl zu entdecken. Dazu werden systematisch verschiedene Bereiche von Kraftquellen vorgestellt, um so die psychische Widerstandskraft (Resilienz) gezielt zu stärken“ (Diegelmann \& Isermann 2011/2012: 8),

(3) „Sie müssen kein anderer Mensch werden; es reicht, wenn Sie mit dem Problem Ihrer überängstlichen Besorgtheit besser umgehen können. [...] Als Autor wünsche ich Ihnen die bestmögliche Umsetzung aller Schritte zu einem Leben mit weniger Ängsten und Sorgen sowie zu mehr Freude und Erfolgserlebnissen. Verzichten Sie dabei auf das unrealistische Ziel eines völlig angst- und sorgenfreien Lebens. Es reicht, wenn Sie trotz gelegentlicher Ängste und Sorgen das tun können, was Ihnen in Ihrem Leben wichtig ist“ (Morschitzky 2017: 8-9).

Die beobachteten Neuerungen greifen über die ersten, einführenden Kapitel hinaus, wie die korpuslinguistischen Analysen zeigen: Nicht alle, aber einige der zentralen Ausdrücke aus älteren Zielformulierungen werden im Laufe der Zeit seltener gebraucht; umgekehrt kommen die oben genannten Ausdrücke aus neueren Zielformulierungen häufiger zum Einsatz (vgl. Abbildungen 3-8). Auch die Ausdrücke Angststörung und Angsterkrankung, die in vielen neueren Ratgebern die angenommene Ausgangssituation bezeichnen, werden mit der Zeit häufiger verwendet (vgl. Abbildungen 9-10). 
fertig werden

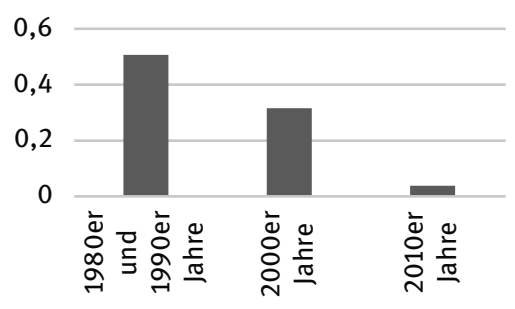

*leid*

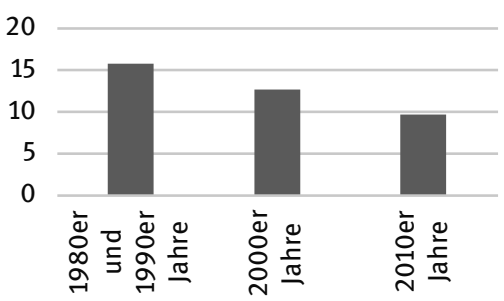

aktivier* $^{*}$

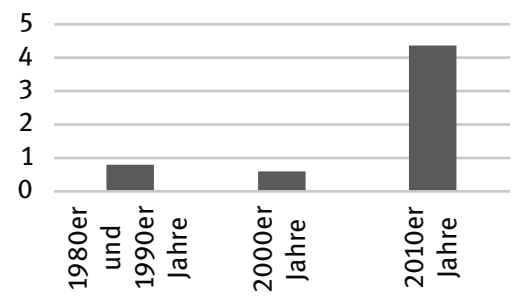

${ }^{*}$ heil ${ }^{*}$

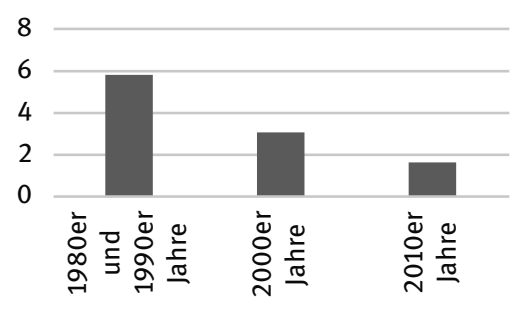

umgehen $\left(\mathrm{VV}^{*}\right)$

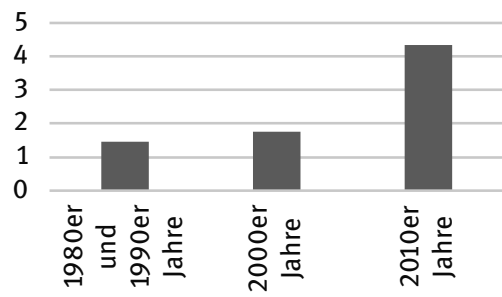

ressource*

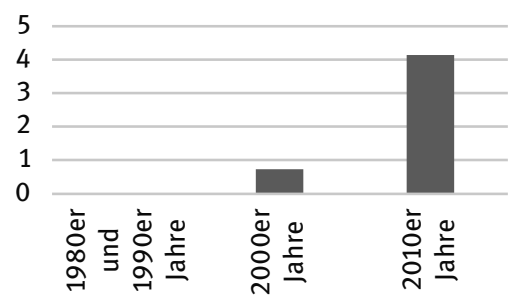

Abb. 3-8: Häufigkeit zentraler Ausdrücke aus Zielformulierungen in den drei digitalen Teilkorpora (Vorkommen pro 10'000 Token)
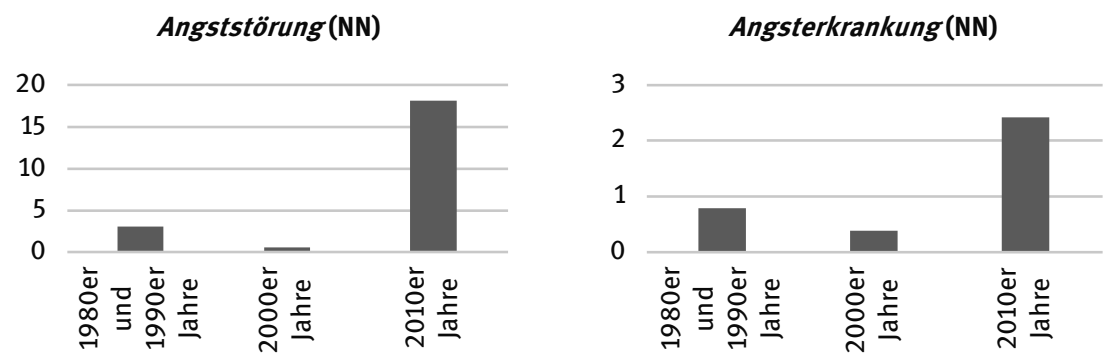

Abb. 9-10: Häufigkeit zentraler Ausdrücke zur Bezeichnung der Ausgangssituation in den drei digitalen Teilkorpora (Vorkommen pro 10’000 Token) 
In den ausgewerteten Ratgebern verändern sich also die Bezeichnung der zentralen Zielgruppe, die Formulierung von Zielen für sie sowie die Benennung ihres Ausgangszustands relativ deutlich, wobei zeitgebundene Muster erkennbar werden.

Wie sind die beschriebenen Veränderungen kulturanalytisch zu interpretieren? Die Veränderung der Bezeichnung von Menschen, die Angst haben, könnte man auf das Bemühen zurückführen, sie möglichst wenig zu stigmatisieren und möglichst wenig als bemitleidenswerte Opfer darzustellen. Solange die neueren Bezeichnungen durch eine vielseitige Verwendung außerhalb der Ratgeber wertneutral bleiben, ist es denkbar, dass dieses Bemühen tatsächlich Erfolg hat.

Dazu scheint auf den ersten Blick im Widerspruch zu stehen, dass die Ratgeber ihrer Hauptzielgruppe immer öfter eine Angststörung oder Angsterkrankung zuschreiben. Erklären lässt sich diese Neuerung damit, dass sich die medizinische bzw. psychiatrische Diagnose von Krankheiten mit Angst verändert hat: Die deutsche Modifikation der zehnten Revision der Internationalen statistischen Klassifikation der Krankheiten und verwandter Gesundheitsprobleme der WHO (ICD-10-GM), die seit dem Jahr 2000 die ,amtliche Klassifikation zur Verschlüsselung von Diagnosen in der ambulanten und stationären Versorgung in Deutschland“ bildet (Deutsches Institut für Medizinische Dokumentation und Information (DIMDI) [o. J.]a), weicht in der Bezeichnung und Einteilung von Angstkrankheiten auffällig stark von der vorausgehenden neunten Revision ab. Kennt die ältere westdeutsche Auflage im Kapitel „V. Psychiatrische Krankheiten“ abgesehen von „Mit Angst und Furchtsamkeit“ einhergehenden „Spezifischen emotionalen Störungen des Kindes- und Jugendalters“ lediglich „Angstneurose“ und „Phobie“ als explizit mit Angst verbundene Diagnosen (Deutsches Institut für Medizinische Dokumentation und Information (DIMDI) [o. J.]b), werden in der neueren Auflage in Kapitel „V Psychische und Verhaltensstörungen“ sowohl verschiedene „Phobische Störungen“ (z. B. „Agoraphobie“, „Soziale Phobien“ oder „Spezifische (isolierte) Phobien“) als auch diverse „Andere Angststörungen“ (z. B. „Panikstörung“ oder „Generalisierte Angststörung“) differenziert und außerhalb dieser beiden Gruppen weitere Diagnosen mit Angstsymptomen (z. B. „Zwangsstörung“ oder „Posttraumatische Belastungsstörung“) genannt (Deutsches Institut für Medizinische Dokumentation und Information (DIMDI) \& World Health Organization (WHO) 1994-2019). Die ICD-10-GM hat damit zweifellos der vermehrten Kategorisierung von Angst als Krankheit sowie auch deren zunehmender Bezeichnung als Störung Vorschub geleistet. Diese Veränderung, die offensichtlich von den untersuchten Ratgebern mitgetragen wird, läuft freilich nicht unbedingt auf eine verstärkte Stigmatisierung von Menschen mit Angst hinaus. Sie kann auch dazu führen, dass 
Menschen, die (zu) viel Angst haben, ähnlich wie Menschen mit (rein) körperlichen Erkrankungen betrachtet werden. Mit großer Wahrscheinlichkeit führt sie zumindest dazu, dass diejenigen, die ihre eigene Angst als problematisch erleben, diese eher als therapierbar ansehen und dementsprechend auch eher nach Behandlungsmöglichkeiten suchen.

Der geschilderte Wandel in den Zielformulierungen schließlich ist vermutlich mit einem umfassenderen medizinischen, psychiatrischen und psychotherapeutischen Trend zur sogenannten salutogenetischen Sichtweise zu begründen, d. h. mit dem zunehmenden Interesse an der Gesunderhaltung und -werdung statt an der Entwicklung und Entstehung von Krankheiten. Das Konzept der Salutogenese wurde 1979 von Aaron Antonovsky als Gegenstück zu dem der Pathogenese entworfen. Indem die untersuchten Angst-Ratgeber sich eine salutogenetische Orientierung zu eigen machen, können sie zur Verbreitung der salutogenetischen Betrachtung und Behandlung von Angst sowie zur Popularisierung der salutogenetischen Perspektive allgemein beitragen.

\subsection{Ratschläge}

Fasst man Ratgeber wie in diesem Beitrag als eine Textsorte auf, in der Anleitungen, Tipps oder - ihrer Bezeichnung entsprechend - Ratschläge gegeben werden, stellt sich natürlich auch die Frage, mit welchen Mitteln diese sprachlichen Handlungen vollzogen werden. Wie wird in den Ratgebern gegen Angst zum Ausdruck gebracht, was man tun soll oder kann, um die eigene Angst als weniger problematisch zu empfinden? Wie in Teil 4.1 erwähnt, enthalten fast alle Ratgeber ein oder mehrere Teile, Kapitel oder Teilkapitel, in dem oder denen Maßnahmen gegen Angst vorgestellt werden, die die/der Lesende allein ergreifen kann. Aus diesen oft umfangreichen Textabschnitten habe ich pro Ratgeber fünf kürzere Passagen, die je eine Maßnahme erläutern, herausgegriffen und exemplarisch auf konkrete Ratschläge und Muster darin untersucht. Unter einem Ratschlag verstehe ich dabei die Empfehlung oder Anregung einer äußerlichen (also sinnlich wahrnehmbaren) oder innerlichen (d. h. mentalen) Handlung, die jemand jemand anderem zur Annäherung an ein bestimmtes Ziel gibt. Mit John Searle (1969/1971: 104-105) gehe ich davon aus, dass Raten oder, synonym dazu, einen Ratschlag Geben ein Typ von illokutionärem Akt ist, der zu den Direktiven gehört. In den untersuchten Ratgebern wird eine Maßnahme gegen Angst in der Regel mit mehreren Ratschlägen erklärt.

Die angeratenen Maßnahmen sind inhaltlich divers, und zum Teil scheinen sie sogar gegenläufig zu sein. Gesamthaft betrachtet setzen sie bei allen drei Dimensionen von Angst an, die in Teil 4.2 angesprochen wurden: bei der menta- 
len Dimension (wiederholt angeraten werden z. B. die genaue Beobachtung der Angst, die Ablenkung davon, das reaktionslose Geschehenlassen der Angst, die Auseinandersetzung mit dem gefürchteten nachteiligen Ereignis und die Verstärkung positiver, vertrauensvoller Einstellungen), bei der körperlichen Dimension (mehrfach empfohlen werden z. B. Entspannungs- bzw. Atemübungen sowie Bewegung bzw. Sport) und bei der das Verhalten betreffenden Dimension (regelmäßig angeregt wird die systematische Konfrontation mit Angstauslösern). Der Veränderung der Zielformulierungen entsprechend, die in Teil 4.3 beschrieben wurde, verlagert sich der Schwerpunkt mit der Zeit in Richtung von Maßnahmen, die Gegenkräfte zu Angst, Stärken und Positives fokussieren.

Was nun die Ratschläge angeht, so zeigen sich in allen betrachteten Jahrzehnten zahlreiche Muster ihrer Formulierung, die zusammen ein größeres Repertoire bilden. Grundlegend zu unterscheiden sind eher explizite und eher implizite Formulierungsmuster: Bei den expliziten Mustern weisen die Formulierungen im Rahmen ihrer grammatischen oder lexikalischen Bedeutungen entweder deontische Komponenten auf, die besagen, was der Fall sein soll, oder aber evaluative Komponenten, die besagen, wie etwas bewertet wird, und die dadurch deontisch lesbar sind (zu diesem Verständnis von deontischer Bedeutung vgl. Hermanns 1989: 74-75). Bei den impliziten Mustern erlaubt es die vorhandene Formulierung, auf eine Aussage zu schließen, die sich mit einem der expliziten Muster verbalisieren ließe. Generell gilt, dass die Zugehörigkeit des jeweiligen Textes zur Textsorte Ratgeber eine entscheidende Rolle dafür spielt, dass die vorhandenen ex- oder implizit direktiven Formulierungsmuster als Ratschläge (und nicht z. B. als Anordnungen, Befehle o. Ä.) aufzufassen sind. Die aufgefundenen Muster bilden Form-Funktions-Einheiten, die sich mit sehr unterschiedlichen Bereichen der bisherigen linguistischen Forschung in Verbindung bringen lassen: Manche expliziten Formulierungsmuster sind als Ausdrucksmittel deontischer Modalität beschrieben worden (zum Begriff der deontischen Modalität vgl. z. B. Palmer 1986/2001: 9-10; Nuyts 2005: 9-10), zur Erfassung anderer expliziter, aber auch impliziter Formulierungsmuster bieten sich im Gegensatz dazu pragmatische und argumentationstheoretische Konzepte an, beispielsweise das des explizit performativen und des indirekten Sprechaktes nach John Austin (vgl. 1962/2002: 88-101) bzw. John Searle (vgl. 1975/1982: 51-79), das der Implikatur von H. Paul Grice (vgl. 1975/1979: 245-255, 262-265) oder das des pragmatischen Arguments im Sinne von Chaïm Perelman und Lucie Olbrechts-Tyteca (vgl. 1958/1971: 266-270). 
Die wichtigsten Muster der expliziten Formulierung von Ratschlägen sind:

I. Konstruktionen mit einem Modalverb

a) mit dem Personalpronomen der ersten Person Plural (inklusiv verwendet):

„Wann immer negative Gefühle oder Empfindungen in uns auftauchen, sollten wir das bemerken und uns Fragen stellen wie: ,Droht eine Gefahr?‘, ,Überanstrenge ich mich?‘, [...]““ (Hansch 2011: 106), „Zunächst müssen wir uns [...] klarmachen, was Gefühle überhaupt sind“ (Illy 2016: 78);

b) mit dem Personalpronomen der dritten Person Plural:

„Wenn Ihr Arzt feststellt, daß Sie körperlich gesund sind, sollten Sie sich durch sportliche Aktivitäten wie regelmäßige Spaziergänge, Schwimmen und andere Sportarten fit halten“ (Wittchen 1997/1999: 59),

„Sie müssen ein Gefühl wie Angst zuerst einmal annehmen lernen“ (Morschitzky 2017: 169);

c) mit dem indefiniten Pronomen man:

„Anstatt zu unterstellen, die Katastrophe stünde unmittelbar bevor, sollte man Informationen sammeln, die dagegen sprechen“ (Schmitz \& Schmitz 2005: 140),

„Man kann Stress reduzieren, bevor er einen erreicht, was leider nicht immer möglich ist. Man kann außerdem an stressverstärkenden Gedanken und Verhaltensweisen arbeiten und zudem eine ausreichende Stresserholung betreiben“ (Illy 2016: 121);

d) mit einer substantivischen Personenbezeichnung oder einem anderen Substantiv:

„Diese Arbeit muß der Patient selbst über neues Denken und dementsprechendes Verhalten in Angriff nehmen“ (Lückert 1993: 107, Hervorheb. i. O.),

„Das erste Ziel sollte eine Situation oder einen Ort betreffen, der relativ wenig Angst einflößt“ (Peurifoy 1997/2007: 125).

II. Konstruktionen mit einem Vollverb im Imperativ bzw. in der Aufforderungsform

a) realisiert in der zweiten Person Singular:

„MERKE Manchmal ist es leider nicht möglich, Belastungen abzubauen. Dann ist es umso wichtiger, mit Entlastungen ein Gegengewicht zu schaffen“ (Wilms \& Wilms 2008: 75), 
„Verändere absichtlich ganz alltägliche Aktivitäten, Denk- und Verhaltensweisen, nicht deine Lebensumstände“ (Diegelmann \& Isermann 2011/2012: 40, als Übersetzung eines englischen Zitats);

b) realisiert in der dritten Person Plural:

„Halten Sie sich täglich ein bißchen Zeit zum Erholen frei“ (Priest 1983/1985: 38),

„Unternehmen Sie verschiedene Verhaltensexperimente leichterer Art und gehen Sie dann zu schwierigeren Aufgabenstellungen über“ (Morschitzky 2017: 132).

III. Konstruktionen mit einem Vollverb im Infinitiv:

„Das innere Kind lieben“ (Trickett 1992/1995: 183, als Überschrift),

„Jede dieser vorsichtigen Annäherungen so lange wiederholen, bis die Angst auszuhalten und ein nächster Schritt zu wagen ist" (Schmitz \& Schmitz 2005: 139).

IV. Explizit performative Ratschläge:

„Das Erlernen dieser Techniken ist im Rahmen einer Angst- oder Depressionstherapie sehr $\mathrm{zu}$ empfehlen“ (Peseschkian \& Boessmann 1998: 144),

„Da Wiederholung der Schlüssel zum Erfolg ist, rate ich Ihnen, dieses Buch ganz langsam und bewusst noch einmal zu lesen“ (Peurifoy 1997/2007: 204).

$V$. Andere explizite evaluative und/oder deontische Formulierungsmuster

a) Überschriften und Vergleichbares:

„Genaue Wahrnehmung der Angst“ (Schmidt-Traub 1995: 46),

„TIPP Eine strukturiertere Selbstbeobachtung hilft, Katastrophengedanken einzudämmen und ermöglicht doch, den Tages- und Wochenrhythmus Ihrer Beschwerden genau zu betrachten“ (Wilms \& Wilms 2008: 74);

b) teilsatzwertige Formulierungen im Fließtext:

„Ein anderes, einfaches Rezept lautet, die Angst weder zu verdrängen noch ihr nachzugeben, sondern sie zwar wahrzunehmen, aber zu ignorieren“ (Schmidbauer 2005: 181, Hervorheb. J.S.),

„Es gilt die Angst so lange auszuhalten und nicht zu vermeiden, bis die Angstgewöhnung eintritt“" (Illy 2016: 112, Hervorheb. J.S.);

c) Einzellexeme im Fließtext:

„Der erste Schritt besteht darin, daß Sie eine neue Einstellung zu Ihrer Angsterkrankung entwickeln“ (Wittchen 1997/1999: 56, Hervorheb. J.S.), 
„Der ideale Zeitpunkt, um zu schreiben, ist unmittelbar nach einer Angstattacke - dann ist die Erinnerung daran noch frisch" (Illy 2016: 87, Hervorheb. J.S.).

$\mathrm{Zu}$ den wichtigsten Mustern der impliziten Formulierung von Ratschlägen gehört, dass eine positiv zu beurteilende Folge der angeratenen Handlung erwähnt wird. Dies geschieht manchmal, aber nicht immer in einem Satzgefüge mit konditionalem Nebensatz. Im Rahmen eines pragmatischen argumentativen Schlusses kann die positive Beurteilung der Folge auf die Ursache übertragen werden, wodurch die jeweilige Handlung als wünschenswert erscheint. Explizit formuliert wird somit eine Prämisse, und impliziert wird damit eine präskriptive Konklusion, der Ratschlag. Beispiele sind etwa:

(1) „Sie kommen besser voran, wenn Sie den Weg der kleinen Schritte einschlagen“ (Schmidt-Traub 1995: 51, Hervorheb. i. O.),

(2) „Viele Ihrer Probleme werden sich bessern oder sogar verschwinden, allein dadurch, dass Sie nicht mehr gegen sie kämpfen, sondern sie akzeptieren“ (Hansch 2011: 26).

Das zweite wichtige implizite Formulierungsmuster besteht in rhetorischen oder reflexionsorientierten Fragen, die den Adressierten nahelegen, was zu tun ist, z. B.:

(1) „Was hilft Ihnen, sich gut zu fühlen? Ein Spaziergang mit dem Hund? Oder ein Vollbad mit einem angenehmen Duft?“ (Wilms \& Wilms 2008: 75),

(2) „Natürlich können Sie dem Chef keine Ohrfeige verpassen, wenn Sie sich über ihn geärgert haben, aber wer hindert Sie daran, nach Feierabend auf einen Box-Sack einzuprügeln?“(Illy 2016: 80).

Das erläuterte Repertoire von Mustern, die sich in den analysierten Ratgebern bei der Formulierung von Ratschlägen zeigen, bleibt von den 1980er bis in die 2010er Jahre stabil. Allerdings variiert die Häufigkeit, mit der die verschiedenen Ratgeber die einzelnen Muster des Repertoires nutzen. Diese Variation in der Frequenz ist sicherlich zum Teil auf individuelle Präferenzen der Autoren zurückzuführen. Inwieweit sie darüber hinaus durch einen historischen Wandel bedingt ist, müssen weitere Untersuchungen prüfen, die den Rahmen dieses Beitrags sprengen würden. 
In den unmittelbaren Kotexten der Ratschläge zeichnen sich allerdings zwei diachrone Veränderungen deutlich ab, die hier noch angesprochen werden können: Sie betreffen beide die sprachliche Gestaltung der Beziehung zwischen der/dem Verfassenden des jeweiligen Ratgebers und der/dem Lesenden (dass diese Beziehung gerade für diachrone Untersuchungen von Ratgebern interessant ist, deutet Niemann 2018: 86 an). Zum einen werden Fragen häufiger, insbesondere solche, die zur Übung gedacht sind bzw. zum Nachdenken anregen sollen, und solche nach den Gedanken oder Gefühlen der Adressierten. Die Fragen dienen weder als Überschriften, noch können sie als indirekte Ratschläge aufgefasst werden:

(1) „Drei Fragen zur Selbstreflexion: In welchen Bereichen bin ich aktiv? Wenn ich mir einen typischen Tagesablauf vorstelle, wie oft mache ich Dinge, die ich wirklich tun will? Welche Zeiten gibt es in meinem Leben, in denen ich mich sehr aktiv gefühlt habe?“ (Diegelmann \& Isermann 2011/2012: 120),

(2) „Vielleicht hatten Sie auch Schwierigkeiten, sich exakt an eine zurückliegende Angstsituation zu erinnern? “ (Illy 2016: 87).

Zum anderen werden Fragen wie die im zweiten gerade zitierten Textausschnitt, aber auch assertive Sprechakte vermehrt mit hedges wie vielleicht, möglicherweise oder bestimmt versehen, die die Gewissheit der Proposition einschränken. Sowohl bei den Fragen wie auch vielfach bei den Assertiven handelt es sich um Sprechakte, die auf die/den Lesende/n referieren. Beispiele für solche Assertive mit einem hedge sind:

(1) „Vielleicht haben Sie auch schon die Idee gehabt, dass Sie es mal mit Entspannungstechniken probieren könnten“ (Wilms \& Wilms 2008: 75),

(2) „Sie haben es bestimmt schon in der letzten Übung gemerkt“ (Illy 2016: 87).

Die beiden Tendenzen zu mehr Fragen und mehr hedges, welche die Gewissheit der jeweiligen Proposition einschränken, lassen sich auch mit korpuslinguistischen Mitteln entdecken. Zum einen werden über die Dekaden hinweg häufiger Fragezeichen verwendet (vgl. Abbildung 11), zum anderen nehmen die Trigramme mit hedges wie den beschriebenen $\mathrm{zu}$ (vgl. Tabelle 4). 


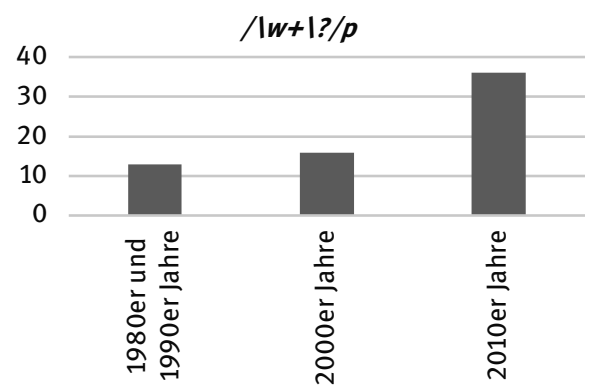

Abb. 11: Häufigkeit von Fragezeichen in den drei digitalen Teilkorpora (Vorkommen pro 10’000 Token)

Tab. 4: Trigramme mit hedges, die die Gewissheit der Proposition einschränken, in den drei digitalen Teilkorpora ${ }^{4}$

\begin{tabular}{|c|c|c|}
\hline $\begin{array}{l}\text { Ratgeber aus den } 1980 \text { er und } \\
1990 \text { er Jahren }\end{array}$ & $\begin{array}{l}\text { Ratgeber aus den 2000er } \\
\text { Jahren }\end{array}$ & $\begin{array}{l}\text { Ratgeber aus den 2010er } \\
\text { Jahren }\end{array}$ \\
\hline- & $\begin{array}{l}\text { kann es sein (18) } \\
\text { dann kann es (7) }\end{array}$ & $\begin{array}{l}\text { kann es hilfreich (10) } \\
\text { sie sich vielleicht (10) } \\
\text { kann es sein (9) } \\
\text { vielleicht haben sie (9) } \\
\text { es ist möglich (7) } \\
\text { kann sein dass (7) }\end{array}$ \\
\hline
\end{tabular}

Die ermittelten Konstanten und Veränderungen in der Formulierung von Ratschlägen und in deren unmittelbarem Kotext eröffnen mehrere Deutungsmöglichkeiten. Zunächst kann man die Anzahl und Art der Formulierungsmuster für Ratschläge in den Angst-Ratgebern schlicht damit erklären, dass sie - im Dienste der Abwechslung und stilistischen Vielfalt - die Formulierungsmöglichkeiten für Ratschläge ausschöpfen, die die zeitgenössische deutsche Sprache generell bietet (vgl. dazu Kessel 2009: 119-134; Ott \& Kiesendahl 2019: 103-106).

Dass viele implizite Ratschläge im Rahmen pragmatischer argumentativer Schlüsse aus den explizit erwähnten positiven Folgen der angeratenen Handlungen zu erschließen sind, bedeutet sodann, dass viele Ratschläge argumentativ begründet werden. Für explizit formulierte Ratschläge werden ebenfalls immer wieder Gründe bzw. Argumente geliefert. Motiviert ist dies möglicher-

4 Es werden nur Trigramme erwähnt, die in einem Teilkorpus mindestens sieben Mal vorkommen. 
weise dadurch, dass die Schreibenden ihre Hauptzielgruppe als oder analog zu ,mündigen Patienten' auffassen: Die Lesenden, die Angst haben, sollen nicht nur etwas tun, sondern auch wissen, warum. Dass die Relation zwischen Rat gebender Person und Rat empfangender Person in den Ratgebertexten in dieser Weise gestaltet wird, bestätigt und verstärkt die Norm des ,mündigen Patienten“ bzw. des ,mündigen Ratempfängers‘.

Die geschilderte Zunahme von Fragen und von hedges schließlich weist in eine ähnliche Richtung: Dass Fragen zunehmen, ließe sich auf den Wunsch nach mehr Ebenbürtigkeit und Dialogizität zwischen den Verfassenden und den Adressierten zurückführen, dass hedges der erwähnten Art häufiger werden, als Ausdruck vermehrter Empathie gegenüber den Angesprochenen und verstärkten Respekts vor unterschiedlichen subjektiven Sichtweisen und individuellen Lebenswirklichkeiten. Mittel- und langfristig können sprachliche Veränderungen wie die geschilderten natürlich nicht nur ein Indikator solch neuer Beziehungsnormen sein, sondern auch einen Einflussfaktor dafür bilden: Sie können dazu beitragen, dass in der Beziehung zwischen Ratgeber und Ratempfänger und möglicherweise auch zwischen Psychotherapeut und Klient mehr Gleichberechtigung bzw. mehr Autonomie und weniger Bevormundung der letztgenannten Partei als üblich gelten und folglich auch erwartet und angestrebt werden.

\section{Fazit}

„Angst ist veraltet und zugleich aktuell“ - mit dieser Formulierung konstatiert Jörg Bergmann (2002: 2, vgl. 2-10), dass Angst auch in der Gegenwart noch eine wichtige Rolle spielt. Er weist auf den merkwürdigen, scheinbar paradoxen Umstand hin, dass umfassende historische Entwicklungen wie der Verlust christlicher Glaubensgewissheiten, der Zuwachs an Wissen oder die verstärkte Konfrontation mit Unbekanntem und Unbekannten verbreiteten Ängsten entgegengewirkt, oft aber zugleich neue Ängste hervorgerufen haben.

Dass Angst auch gegenwärtig noch sehr präsent ist, ließe sich vor dem Hintergrund dieser Studie allerdings auch anders begründen: Wie oben beschrieben, setzt Angst die Antizipation von Nachteiligem voraus, wie diffus dieses Nachteilige im Einzelfall auch erscheinen mag. Zwar sind bedeutende historische Lebensrisiken, etwa das Risiko, lebensbedrohlich zu erkranken, einen Krieg zu erleben oder von existenzieller Armut betroffen zu sein, im deutschsprachigen Raum der Tendenz nach gesunken. Doch je mehr ,jeder Einzelne [...] von klein auf dazu gedrängt [wird], die Wirkung seiner Handlungen oder die Wirkung der Handlungen von Anderen über eine ganze Reihe von Kettenglie- 
dern hinweg zu bedenken“, wie Norbert Elias eine zentrale Entwicklung im „Prozeß der Zivilisation“ beschreibt, je mehr „Training zur Langsicht“ also stattfindet (Elias 1939/1978: 2. Bd.: 322, 341), desto stärker ist sie/er nicht nur zur Antizipation generell, sondern wohl auch speziell zur Antizipation von Nachteiligem und also zur Angst disponiert.

Die Ratgeber gegen Angst, die ich hier untersucht habe, offenbaren sich in der Rückschau und Reflexion auf die vorgestellten Ergebnisse in zweierlei Hinsicht als janusgesichtiges, ambivalentes Kulturphänomen: Zum einen wollen die Ratgeber ihrer Leserschaft zwar dazu verhelfen, die eigene Angst als weniger problematisch $\mathrm{zu}$ empfinden. Mit der dazu notwendigen Kommunikation über Angst rücken sie diese aber zwangsläufig in den Fokus der Aufmerksamkeit, und sie können nicht verhindern, ihre Leserschaft dadurch für die Empfindung von Angst zusätzlich anfällig zu machen. Zum anderen sind die Ratgeber darauf angelegt, neue Einstellungs- und Handlungsmöglichkeiten bei bzw. gegenüber Angst aufzeigen. Doch als appellative, instruktive Textsorte setzen und verfestigen sie auch automatisch emotionale Standards. Sie legen feeling rules im Sinne Hochschilds fest und verstärken sie, sie tragen zu emotionology im Verständnis von Stearns und Stearns bei. Ihr emanzipatorischer Anspruch wird also unvermeidlich von ihrer für Angst sensibilisierenden und diese normierenden Wirkung konterkariert.

Die zuletzt angesprochene, emotional normierende Wirkung der Ratgeber entspringt wesentlich aus ihrer Kernaussage und deren Präsuppositionen. Die Kernaussage lässt sich im Rückblick auf die vorgestellten expliziten sprachlichen Muster so zusammenfassen: Gegen Ihre Angst sollten Sie mit den Maßnahmen $X, Y, Z$ vorgehen. Zum Abschluss dieses Beitrags sollen die wichtigsten Präsuppositionen dieser Kernaussage diskutiert werden, die bislang noch nicht voll zur Sprache kamen.

In der genannten Kernaussage und dementsprechend in den Ratgebern generell erscheint Angst vorrangig als individuelle und kaum als kollektive Emotion oder, präziser, als soziales oder gesellschaftliches Phänomen. Wie in Teil 4.2 deutlich wurde, wird normalerweise vorausgesetzt, dass Angst das emotionale Erleben einer/eines Einzelnen ist. Diese Perspektive hat zur Folge, dass auch für den Umgang mit Angst nur das Individuum und keine Gruppe oder gar Gesellschaft als zuständig erscheint und dass zudem als mögliche Ursachen von Angst eher persönliche als soziale oder gesellschaftliche Faktoren in den Blick geraten. Mit ihrer Kernaussage präsupponieren die Ratgeber zweitens, dass die Adressierten derzeit zu viel Angst empfinden und dass diese weniger dominant werden soll und kann. Dass Angst nicht einfach als menschliche Existenzbedingung ertragen oder ausgehalten werden muss, sondern dass sie, wie in Teil 
4.3 beschrieben, aktiv zu reduzieren oder mithilfe anderer Gefühle zu entkräften ist, lässt sich somit als die zentrale feeling rule der Ratgeber festhalten. You shouldn't feel so anxious, kann sie in Abwandlung eines Hochschild'schen Beispiels formuliert werden (vgl. Hochschild 1979: 564). Drittens schließlich unterstellen die Ratgeber mit ihrer Kernaussage, dass jemand, die/der zu viel Angst hat, diese selbstständig regulieren soll und kann, ohne dabei auf die persönliche Hilfe einer/eines anderen angewiesen zu sein. Dadurch wird $\mathrm{zu}$ emotion work in Form der Selbsthilfe oder Selbststeuerung animiert, wie in Teil 4.4 ersichtlich wurde. Auch für den Umgang mit Angst gilt folglich der Imperativ der Selbstoptimierung, der sich so gut in das von Ulrich Bröckling beschriebene „Subjektivierungregime“ des „unternehmerischen Selbst“ einfügt (Bröckling 2007: 13). Damit changieren die Ratgeber zwischen der Ermächtigung der/des Einzelnen zur Einwirkung auf die eigenen Gefühle und ihrer/seiner Belastung mit der Verantwortung, die eigenen Gefühle so zu regeln, dass sie der von ihnen gesetzten sozialen Norm entsprechen.

Spätestens mit diesen abschließenden Überlegungen wird deutlich, wie reichhaltig Ratgeber gegen Angst für linguistische und kulturanalytische Studien sind. Dementsprechend bieten sich zahlreiche weitere Untersuchungen an: So könnten Angst-Ratgeber etwa hinsichtlich zusätzlicher sprachlicher Muster, z. B. bei der Beschreibung von Angstursachen oder mit Blick auf die Vermittlung von Fachwissen, analysiert werden. Weiterhin wäre der Sprachgebrauch in anderen Texten und Gesprächen, in denen Angst thematisiert wird, beispielsweise in wissenschaftlichen oder in Fachtexten, idealerweise aber auch in Therapiegesprächen, mit dem der Ratgeber zu vergleichen. Außerdem läge es nahe, die Versprachlichung anderer Emotionen in Bezug zur Versprachlichung von Angst in Ratgebern und anderen Kontexten zu setzen. Und bei all dem wären schließlich zum einen diachrone Untersuchungen und zum anderen Vergleiche mit anderen Sprachen denkbar. Erst mit einer ganzen Reihe solcher Studien ließen sich unsere Vorstellungen davon, was Angst ist und was man mit ihr tun kann oder soll, wirklich verstehen, und es ließe sich erkennen, inwiefern diese Vorstellungen sowohl kulturell geprägt als auch kulturell prägend sind.

\section{Literatur}

\section{Quellen}

Craske, Michelle G. \& David H. Barlow (2006/2016): Meistern Sie Angst und Sorgen! Generalisierte Angststörung bewältigen. Ein Patientenmanual. Hrsg., übs. und adapt. von Christoph Flückiger. Bern: Hogrefe. 
Diegelmann, Christa \& Margarete Isermann (2011/2012): Kraft in der Krise. Ressourcen gegen die Angst. 2. Aufl. Stuttgart: Klett-Cotta.

Hansch, Dietmar (2011): Erfolgreich gegen Depression und Angst. Berlin: Springer.

Heidegger, Martin (1927/1977): Sein und Zeit. 14., durchges. Aufl. Tübingen: Niemeyer.

Hoffmann, Walter (2007): Kraftquelle Angst. So nutzen Sie Ihr Frühwarnsystem. Wien: Ueberreuter.

Illy, Daniel (2016): Ratgeber Angsterkrankungen. Hilfe für den Alltag. München: Urban und Fischer.

Kierkegaard, Sören (1844/2005): Der Begriff Angst. Übs. von Hans Rochol. Hamburg: Meiner.

Lückert, Heinz-Rolf (1993): Angst und Panik. Ursachen, Symptome, Therapie. Niedernhausen im Taunus: Falken.

Morschitzky, Hans (2017): Angst und Sorgen die Macht nehmen. Selbsthilfe bei Generalisierter Angststörung. Ostfildern: Patmos.

Peiffer, Vera (2004): Nur keine Angst! Das Erfolgsprogramm gegen Angstzustände und Panikattacken. Wirksame Techniken zur Selbsthilfe. München: Knaur.

Peseschkian, Nossrat \& Udo Boessmann (1998): Angst und Depression im Alltag. Eine Anleitung zu Selbsthilfe und positiver Psychotherapie. Frankfurt am Main: FischerTaschenbuch.

Peurifoy, Reneau Z. (1997/2007): Frei von Angst - ein Leben lang. Hilfe zur Selbsthilfe. Übs. von Irmela Erckenbrecht. Bern: Huber.

Priest, Robert (1983/1985): Angst und Depressionen. Ein praktischer Ratgeber. Übs. von Susanne Wagner. Wien: Orac.

Schmidbauer, Wolfgang (2005): Lebensgefühl Angst. Jeder hat sie. Keiner will sie. Was wir gegen Angst tun können. Freiburg im Breisgau: Herder.

Schmidt-Traub, Sigrun (1995): Angst bewältigen. Selbsthilfe bei Panik und Agoraphobie. Berlin: Springer.

Schmitz, Margot \& Michael Schmitz (2005): Seelenfraß. Wie Sie den inneren Terror der Angst besiegen. [...]. Wien: Ueberreuter.

Trickett, Shirley (1992/1995): Angstzustände und Panikattacken erfolgreich meistern. Übs. von Erna Tom. Zürich: Oesch.

Voos, Dunja (2015): Die eigene Angst verstehen. Ein Ratgeber. Gießen: Psychosozial.

Wilms, Bettina \& Hans-Ulrich Wilms (2008): Meine Angst. Eine Krankheit? Bonn: Balance.

Wittchen, Hans-Ulrich (1997/1999): Wenn Angst krank macht. Störungen erkennen, verstehen und behandeln. München: Mosaik.

\section{Forschungsliteratur}

Antos, Gerd (1996): Laien-Linguistik. Studien zu Sprach- und Kommunikationsproblemen im Alltag. Am Beispiel von Sprachratgebern und Kommunikationstrainings. Tübingen: Niemeyer.

Athanasiadou, Angeliki \& Elżbieta Tabakowska (Hrsg.) (1998): Speaking of emotions. Conceptualisation and expression. Berlin: De Gruyter.

Austin, John L. (1962/2002): Zur Theorie der Sprechakte. (How to do things with Words). Bearb. von Eike von Savigny. 2., erg. Aufl. Stuttgart: Reclam. 
Bergenholtz, Henning (1980): Das Wortfeld „Angst“. Eine lexikographische Untersuchung mit Vorschlägen für ein großes interdisziplinäres Wörterbuch der deutschen Sprache. Stuttgart: Klett-Cotta.

Bergmann, Jörg (2002): Paradoxien der Angstkommunikation. Über Veralten und Modernität der Angst. Jahrbuch für Gruppenanalyse und ihre Anwendungen 8, 1-13.

Bourke, Joanna (2005/2006): Fear. A cultural history. London: Virago.

Bremerich-Vos, Albert (1991): Populäre rhetorische Ratgeber. Historisch-systematische Untersuchungen. Tübingen: Niemeyer.

Brezina, Vaclav, Tony McEnery \& Stephen Wattam (2015): Collocations in context. A new perspective on collocation networks. International journal of corpus linguistics 20 (2), 139173.

Bröckling, Ulrich (2007): Das unternehmerische Selbst. Soziologie einer Subjektivierungsform. Frankfurt am Main: Suhrkamp.

Cieślarová, Eva (2012): Konzeptualisierung der Emotion Angst in deutschen und tschechischen Phraseologismen. Studia Germanistica. Acta facultatis philosophicae universitatis ostraviensis (11), 5-23.

Dobrovol'skij, Dmitrij (1995): Schiß und Espenlaub. Idiome der Angst. Folia Linguistica. Acta societatis linguisticae europaeae 29 (3-4), 317-346.

Elias, Norbert (1939/1978): Über den Prozeß der Zivilisation. Soziogenetische und psychogenetische Untersuchungen. 2 Bde. 5. Aufl. Frankfurt am Main: Suhrkamp.

Ettl, Susanne (1984): Anleitungen zu schriftlicher Kommunikation. Briefsteller von 1880 bis 1980. Tübingen: Niemeyer.

Fiehler, Reinhard (2001): Emotionalität im Gespräch. In: Klaus Brinker, Gerd Antos, Wolfgang Heinemann et al. (Hrsg.), Text- und Gesprächslinguistik. Ein internationales Handbuch zeitgenössischer Forschung. 2. Bd., 1425-1438. Berlin: De Gruyter.

Filatkina, Natalia (2016): Darumb kam eine seer grosse furcht vnd schrecken in das Volck. angest vs. vorhte. Martin Luther als Wendepunkt? In: Nina Bartsch \& Simone SchultzBalluff (Hrsg.), Perspektivwechsel. Oder: Die Wiederentdeckung der Philologie. 2. Bd.: Grenzgänge und Grenzüberschreitungen. Zusammenspiele von Sprache und Literatur in Mittelalter und Früher Neuzeit, 67-89. Berlin: Schmidt.

Filatkina, Natalia (2015): Diskurshistorische Analysen des Begriffs Zukunftsangst anhand des Spiegel-Online-Archivs. Sprachwissenschaft 40 (1), 73-126.

Georgi, Christopher (2018): Zur sprachlichen Thematisierung der Angst in Folge von Terrorismus. Eine datengeleitete Studie. In: Fabian Klinker, Joachim Scharloth \& Joanna Szczęk (Hrsg.), Sprachliche Gewalt. Formen und Effekte von Pejorisierung, verbaler Aggression und Hassrede, 109-134. Stuttgart: Metzler.

Gkonou, Christina, Mark Daubney \& Jean-Marc Dewaele (Hrsg.) (2017): New insights into language anxiety. Theory, research and educational implications. Bristol: Multilingual matters.

Gredel, Eva (2016): „Wenn der Körper ausbrennt“. Burn-out: Sprachliche und semiotische Strategien zur Aushandlung eines Krankheitsbildes. In: Eglè Kontutytė \& Vaiva Žeimantienè (Hrsg.), Sprache in der Wissenschaft. Germanistische Einblicke, 221-235. Frankfurt am Main: Lang.

Grice, H. Paul (1975/1979): Logik und Konversation. Übs. von A.[ndreas] Kemmerling. In: Georg Meggle (Hrsg.), Handlung, Kommunikation, Bedeutung, 243-265. Frankfurt am Main: Suhrkamp. 
Gülich, Elisabeth, Katrin Lindemann \& Martin Schöndienst (2010): Interaktive Formulierung von Angsterlebnissen im Arzt-Patient-Gespräch. Eine Einzelfallstudie. In: Ulrich Dausendschön-Gay, Christine Domke \& Sören Ohlhus (Hrsg.), Wissen in (Inter-)Aktion. Verfahren der Wissensgenerierung in unterschiedlichen Praxisfeldern, 135-160. Berlin: De Gruyter.

Günthner, Susanne (2006): Rhetorische Verfahren bei der Vermittlung von Panikattacken. Zur Kommunikation von Angst in informellen Gesprächskontexten. Gesprächsforschung. Online-Zeitschrift zur verbalen Interaktion 7, 124-151.

Guntersdorfer, Ivett R. (2013): Angst aus der Perspektive der Psychologie bei Arthur Schnitzler und Christa Wolf. Würzburg: Königshausen und Neumann.

Hermanns, Fritz (1989): Deontische Tautologien. Ein linguistischer Beitrag zur Interpretation des Godesberger Programms (1959) der Sozialdemokratischen Partei Deutschlands. In: Josef Klein (Hrsg.), Politische Semantik. Bedeutungsanalytische und sprachkritische Beiträge zur politischen Sprachverwendung, 69-149. Opladen: Westdeutscher.

Hochschild, Arlie R. (1979): Emotion work, feeling rules, and social structure. American journal of sociology 85 (3), 551-575.

lakushevic, Marina (2018): Kollokationen in populärwissenschaftlichen Texten am Beispiel des Depression-Diskurses. In: Laurent Gautier (Hrsg.), Le figement en discours spécialisé, 111130. Berlin: Frank und Timme.

Käuser, Andreas (2013): Medienkulturen der Angst. Einleitung: Angst, Medialität und Repräsentation. In: Lars Koch (Hrsg.), Angst. Ein interdisziplinäres Handbuch, 141-147. Stuttgart: Metzler.

Kessel, Katja (2009): Die Kunst des Smalltalks. Sprachwissenschaftliche Untersuchungen zu Kommunikationsratgebern. Tübingen: Narr.

Knerich, Heike (2013): Vorgeformte Strukturen als Formulierungsressource beim Sprechen über Angst und Anfälle. Berlin: Logos.

Knuchel, Daniel \& Janine Luth ([erscheint]): Sprache und Angst. Interdisziplinäre und linguistische Perspektiven.

Laffan, Michael \& Max Weiss (Hrsg.) (2012): Facing fear. The history of an emotion in global perspective. Princeton: Princeton Univ. Press.

Lindemann, Katrin (2012): Angst im Gespräch. Eine gesprächsanalytische Studie zur kommunikativen Darstellung von Angst. Göttingen: V\&R unipress.

Linke, Angelika (2011): Signifikante Muster. Perspektiven einer kulturanalytischen Linguistik. In: Elisabeth Wåghäll Nivre, Brigitte Kaute, Bo Andersson et al. (Hrsg.), Begegnungen. Das 8. Nordisch-baltische Germanistentreffen in Sigtuna vom 11. bis zum 13.6.2009, 23-44. Stockholm: Stockholm Univ.

Linke, Angelika (1996): Sprachkultur und Bürgertum. Zur Mentalitätsgeschichte des 19. Jahrhunderts. Stuttgart: Metzler.

Nickisch, Reinhard M. G. (1969): Die Stilprinzipien in den deutschen Briefstellern des 17. und 18. Jahrhunderts. Göttingen: Vandenhoeck und Ruprecht.

Niemann, Robert (2020): Zum Wandel des wissenschaftlichen Subjekts. Von kritischer Wissensschöpfung zum postkritischen Selbstmanagement? Bielefeld: Transcript.

Niemann, Robert (2018): Ratgeberliteratur und Strategien der Normierung. Am Beispiel von Wissenschaftsratgebern. In: Beata Mikołajczyk, Michat Piosik, Janusz Taborek et al. (Hrsg.), Studien zur deutschen Grammatik. In memoriam Józef Darski (1941-2016), 75-88. Poznań: UAM. 
Nuyts, Jan (2005): The modal confusion. On terminology and the concepts behind it. In: Alex Klinge \& Henrik H. Müller (Hrsg.), Modality. Studies in form and function, 5-38. London: Equinox.

Ott, Christine \& Jana Kiesendahl (2019): Ratgeber und RAT GEBEN. Textlinguistische Reflexionen zur Identifizierung, Typologisierung und pragmatisch-stilistischen Analyse von Ratgebern. In: Michaela Schmid, Ulf Sauerbrey \& Steffen Großkopf (Hrsg.), Ratgeberforschung in der Erziehungswissenschaft. Grundlagen und Reflexionen, 79-115. Bad Heilbrunn: Klinkhardt.

Palmer, F.[rank] R. (1986/2001): Mood and modality. 2. Aufl. Cambridge: Cambridge Univ. Press.

Perelman, Ch.[aïm] \& L.[ucie ] Olbrechts-Tyteca (1958/1971): The new rhetoric. A treatise on argumentation. Übs. von John Wilkinson \& Purcell Weaver. Notre Dame: Univ. of Notre Dame Press.

Plamper, Jan \& Benjamin Lazier (Hrsg.) (2012): Fear. Across the disciplines. Pittsburgh: Univ. of Pittsburgh Press.

Scheidt, Carl E., Gabriele Lucius-Hoene \& Anja Stukenbrock et al. (Hrsg.) (2015): Narrative Bewältigung von Trauma und Verlust. Stuttgart: Schattauer.

Schneider, Jan G. (2005): Was ist ein sprachlicher Fehler? Anmerkungen zu populärer Sprachkritik am Beispiel der Kolumnensammlung von Bastian Sick. Aptum. Zeitschrift für Sprachkritik und Sprachkultur (2), 154-177.

Schröter, Juliane, Susanne Tienken \& Yvonne Ilg (2019): Linguistische Kulturanalyse. Eine Einführung. In: Juliane Schröter, Susanne Tienken \& Yvonne Ilg et al. (Hrsg.), Linguistische Kulturanalyse, 1-27. Berlin: De Gruyter.

Schröter, Juliane (2014): Analyse von Sprache als Analyse von Kultur. Überlegungen zur kulturanalytischen Linguistik am Beispiel des Wandels von Briefschlüssen im 19. und 20. Jahrhundert. In: Nora Benitt, Christopher Koch \& Katharina Müller et al. (Hrsg.), Kommunikation - Korpus - Kultur. Ansätze und Konzepte einer kulturwissenschaftlichen Linguistik, 2545. Trier: WVT.

Schröter, Juliane (2011): Offenheit. Die Geschichte eines Kommunikationsideals seit dem 18. Jahrhundert. Berlin: De Gruyter.

Schuster, Britt-Marie (2010): Auf dem Weg zur Fachsprache. Sprachliche Professionalisierung in der psychiatrischen Schreibpraxis (1800-1939). Berlin: De Gruyter.

Schwarz, Alexander (1988): Keine Angst in der Sprachwissenschaft. In: Hans-Jürg Braun \& Alexander Schwarz (Hrsg.), Angst, 83-92. Zürich: VDF.

Schwarz-Friesel, Monika (2007/2013): Sprache und Emotion. 2., aktual. und erw. Aufl. Tübingen: Francke.

Searle, John R. (1975/1982): Indirekte Sprechakte. In: John Searle, Ausdruck und Bedeutung. Untersuchungen zur Sprechakttheorie. Übs. von Andreas Kemmerling, 51-79. Frankfurt am Main: Suhrkamp.

Searle, John R. (1969/1971): Sprechakte. Ein sprachphilosophischer Essay. Übs. von R.[enate] und R.[olf] Wiggershaus. Frankfurt am Main: Suhrkamp.

Slunecko, Thomas (2009/2017): Psychotherapie. Eine Lagebestimmung. In: Thomas Slunecko (Hrsg.), Psychotherapie. Eine Einführung. 2., überarb. Aufl., 11-32. Wien: Facultas.

Stearns, Peter N. \& Carol Z. Stearns (1985): Emotionology. Clarifying the history of emotions and emotional standards. The American historical review 90 (4), 813-836.

Walton, Douglas (2000): Scare tactics. Arguments that appeal to fear and threats. Dordrecht: Kluwer Academic. 
Wandruszka, Mario (1950/1981): Angst und Mut. 2. Aufl. Stuttgart: Klett-Cotta.

Wodak, Ruth (2015): The politics of fear. What right-wing populist discourses mean. London:

Sage.

\section{Sonstiges}

Asendorpf, Jens B. \& Franz Caspar (2019): Angst. In: Dorsch. Lexikon der Psychologie. https://portal-hogrefe-com.ezproxy.uzh.ch/dorsch/angst-1/ (8. März 2021).

Bibliographisches Institut (2019a): Angst. In: Duden. Wörterbuch. https://www.duden.de/rechtschreibung/Angst (8. März 2021).

Bibliographisches Institut (2019b): Ratgeber. In: Duden. Wörterbuch. https://www.duden.de/rechtschreibung/Ratgeber (8. März 2021).

Brezina, Vaclav, Matt Timperley \& Tony McEnery (2018): \#LancsBox. Version 4.0. http://corpora.lancs.ac.uk/lancsbox/ (8. März 2021).

Deutsche Nationalbibliothek (Hrsg.) (2019): Katalog der Deutschen Nationalbibliothek. https://portal.dnb.de/opac.htm?method=simpleSearch\&query (8. März 2021).

Deutsches Institut für Medizinische Dokumentation und Information (DIMDI) \& World Health Organization (WHO) (1994-2019): Internationale statistische Klassifikation der Krankheiten und verwandter Gesundheitsprobleme. 10. Revision. German Modification. Version 2019.

https://www.dimdi.de/static/de/klassifikationen/icd/icd-10-gm/kodesuche/htmlgm2019/ (8. März 2021).

Deutsches Institut für Medizinische Dokumentation und Information (DIMDI) ([o. J.]a): ICD-10GM.

https://www.dimdi.de/dynamic/de/klassifikationen/icd/icd-10-gm/ (8. März 2021).

Deutsches Institut für Medizinische Dokumentation und Information (DIMDI) (Hrsg.) ([o. J.]b): ICD-9. Internationale Statistische Klassifikation der Krankheiten, Verletzungen und Todesursachen. 9. Revision.

https://www.dimdi.de/dynamic/de/klassifikationen/icd/icd-10-who/historie/icdvorgaenger/icd-9/icd-9-vas/ (8. März 2021).

Digitales Wörterbuch der deutschen Sprache (2019): Betroffene. In: DWDS-Wortprofil. https://dwds.de/wp/Betroffene (8. März 2021).

Dornseiff, Franz \& Uwe Quasthoff (1934/2004): Der deutsche Wortschatz nach Sachgruppen. 8., neu bearb. Aufl. Berlin: De Gruyter.

Infocenter der R+V-Versicherung (2019): Die Ängste der Deutschen 2019. www.die-aengste-der-deutschen.de (8. März 2021).

Kluge, Friedrich \& Elmar Seebold (1883/2012): Angst. In: Etymologisches Wörterbuch der deutschen Sprache.

https://www-degruyter-com.uaccess.univie.ac.at/view/Kluge/kluge.422?rskey=col0xz\&result=1\&dbq_0=Angst\&dbf_0=kluge-fulltext\&dbt_0=fulltext\&o_0=AND (8. März 2021).

Robert Koch-Institut (Hrsg.) (2015): Gesundheit in Deutschland. https://www.rki.de/-

$\mathrm{DE} /$ Content/Gesundheitsmonitoring/Gesundheitsberichterstattung/GesInDtld/gesundhe it_in_deutschland_2015.html;jsessionid=C7ACD35EAEA81C5F7839531DFA79D77B.interne t082?nn=2379316 (8. März 2021). 